\title{
Ellipsometry in Interface Science
}

\author{
H. Motschmann and R. Teppner \\ Max-Planck-Institut für Grenzflächenforschung, Golm
}

\section{Contents}

1 What is it all about? $\quad 2$

2 Polarized light 2

3 Basic equation of ellipsometry 5

4 Design of an ellipsometer $\quad 6$

5 Theory of reflection $\quad 10$

$6 \quad$ Ellipsometry applied to ultrathin films $\quad 17$

$\begin{array}{lll}7 & \text { Microscopic model for reflection } & 19\end{array}$

8 Adsorption layers of soluble surfactants $\quad 23$

8.1 Importance of purification . . . . . . . . . . . . . . . . 23

8.2 Physicochemical properties of our model systems . . . . . . . . . . 23

8.3 Adsorption layer of a nonionic surfactant . . . . . . . . . . . . . . . . 24

8.4 Ionic surfactant at the air-water interface . . . . . . . . . . . . . . . 25

8.5 Kinetics of absorption . . . . . . . . . . . . . . . 34

9 Principle of imaging ellipsometry $\quad 36$

9.1 Depth of field problem . . . . . . . . . . . . . . . . 37

9.2 Beyond the diffraction limit . . . . . . . . . . . . . . . . . . 39 


\section{What is it all about?}

The following chapter presents the basics of ellipsometry and discusses some recent advances. The article covers the formalism and theory used for data analysis as well as instrumentation. The treatment is also designed to familiarize newcomers to this field. The experimental focus is on adsorption layers at the air-water and oil-water interface. Selected examples are discussed to illustrate the potential as well as the limits of this technique. The authors hope, that this article contributes to a wider use of this technique in the colloidal physics and chemistry community. Many problems in our field of science can be tackled with this technique.

Ellipsometry refers to a class of optical experiments which measure changes in the state of polarization upon reflection or transmission on the sample of interest. It is a powerful technique for the characterization of thin films and surfaces. In favorable cases thicknesses of thin films can be measured to within $\AA$ accuracy, furthermore it is possible to quantify submonolayer surface coverages with a resolution down to $1 / 100$ of a monolayer or to measure the orientation adopted by the molecules on mesoscopic length scales. The high sensitivity is remarkable if one considers that the wavelength of the probing light is on the order of $500 \mathrm{~nm}$. The data accumulation is fast and allows to monitor the kinetics of adsorption processes. The technique can also be extended to a microscopy. Imaging ellipsometry allows under certain conditions a direct visualization of surface inhomogeneities as well as quantification of the images. Many samples are suitable for ellipsometry and the only requirement is that they must reflect laser light. Its simplicity and power makes ellipsometry an ideal surface analytical tool for many objects in interface science.

\section{Polarized light}

Light is an electromagnetic wave and all its features relevant for ellipsometry can be described within the framework of Maxwell's theory [1]. The relevant material properties are described by the complex dielectric function $\epsilon$ or alternatively by the corresponding refractive index $n$. 
An electromagnetic wave consists of an electric field $\vec{E}$ and a magnetic field $\vec{B}$. The field vectors are mutually perpendicular and also perpendicular to the propagation direction as given by the wave vector $\vec{k}$. All states of polarization are classified according to the trace of the electrical field vector during one period. Linearly polarized light means the electrical field vector oscillates within a plane, elliptically polarized light means that the trace of the electric field vector during one period is an ellipse. A convenient mathematical representation of a given state of polarization is based on a superposition of two linearly polarized light waves within an arbitrarily chosen orthogonal coordinate system.

$$
\vec{E}(\vec{r}, t)=\left(\begin{array}{c}
\left|E_{\mathrm{p}}\right| \cos \left(2 \pi \nu t-\vec{k} \cdot \vec{r}+\delta_{\mathrm{p}}\right) \\
\left|E_{\mathrm{s}}\right| \cos \left(2 \pi \nu t-\vec{k} \cdot \vec{r}+\delta_{\mathrm{s}}\right)
\end{array}\right)
$$

$\left|E_{\mathrm{p}}\right|$ and $\left|E_{\mathrm{s}}\right|$ are the amplitudes, $\delta_{\mathrm{p}}$ and $\delta_{\mathrm{s}}$ the phases, $|\vec{k}|=2 \pi / \lambda$ is the magnitude of the wave vector and $\nu$ the frequency. Only the phases $\delta_{\mathrm{p}}, \delta_{\mathrm{s}}$ and the amplitudes are required for a representation of the state of polarization; the time dependence is not of importance and can be neglected. The so called Jones vector reads

$$
\vec{E}=\left(\begin{array}{c}
\left|E_{\mathrm{p}}\right| e^{i \delta_{\mathrm{p}}} \\
\left|E_{\mathrm{s}}\right| e^{i \delta_{\mathrm{s}}}
\end{array}\right)=\left(\begin{array}{c}
E_{\mathrm{p}} \\
E_{\mathrm{s}}
\end{array}\right)
$$

Different states of polarization are depicted in Fig. 1. The state of polarization is

a) linear, if $\delta_{\mathrm{p}}-\delta_{\mathrm{s}}=0$ or $\delta_{\mathrm{p}}-\delta_{\mathrm{s}}=\pi$,

b) elliptical, if $\delta_{\mathrm{p}} \neq \delta_{\mathrm{s}}$ and $\left|E_{\mathrm{p}}\right| \neq\left|E_{\mathrm{s}}\right|$,

c) circular for the special case $\delta_{\mathrm{p}}-\delta_{\mathrm{s}}=\pi / 2$ and $\left|E_{\mathrm{p}}\right|=\left|E_{\mathrm{s}}\right|$.
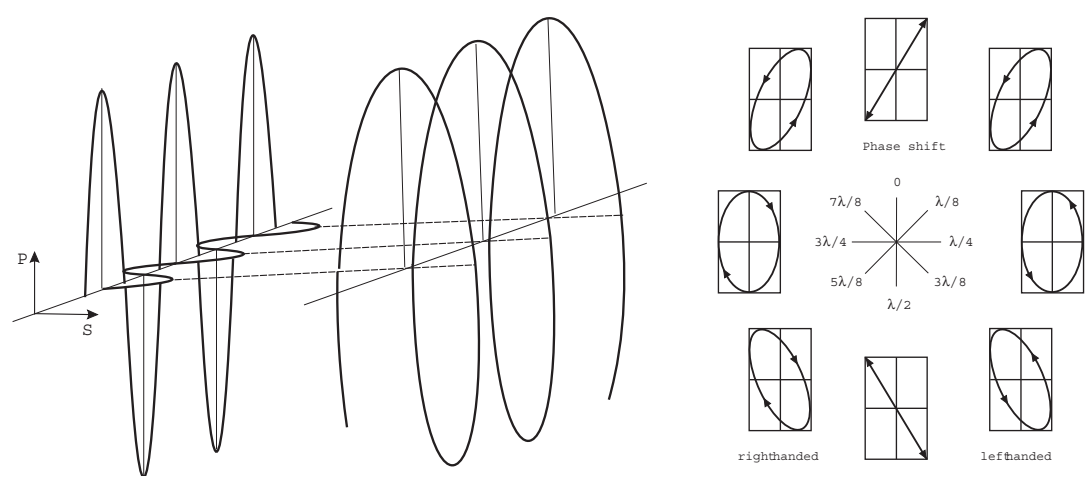

Figure 1: The Jones representation of polarized light represents any state of polarization as a linear combination of two orthogonal linearly polarized light waves. 
An alternative representation of a given state of polarization uses the quantities ellipticity $\omega$ and azimuth $\alpha$. The ellipticity is defined as the ratio of the length of the semi-minor axis to that of the semi-major axis as shown in Fig. 2. The azimuth angle $\alpha$ is measured counterclockwise from the $\hat{x}$-axis. Light is assumed to propagate in positive $\hat{z}$ direction so that $\hat{x}, \hat{y}$ and $\hat{z}$ define a right handed coordinate system. Some authors prefer this representation and for this reason the conversion formulas between both notations are listed below. The derivations require a vast number of tedious algebraic manipulations and can be found in $[2]$.

$$
\begin{aligned}
& \tan 2 \alpha=\frac{2 E_{x} E_{y} \cos \left(\delta_{y}-\delta_{x}\right)}{E_{x}^{2}-E_{y}^{2}}-90^{\circ} \leq \alpha \leq 90^{\circ} \\
& \sin (2 \omega)=\frac{2 E_{x} E_{y} \sin \left(\delta_{y}-\delta_{x}\right)}{E_{x}^{2}+E_{y}^{2}}-45^{\circ} \leq \omega \leq 45^{\circ} \\
& \tan \left(\delta_{y}-\delta_{x}\right)=\frac{\tan (2 \omega)}{\sin (2 \alpha)} \quad-180^{\circ} \leq \delta_{y}-\delta_{x} \leq 180^{\circ} \\
& \cos \left(2 \arctan \left(\frac{E_{y}}{E_{x}}\right)\right)=\cos (2 \omega) \cos (2 \alpha) \quad 0^{\circ} \leq \arctan \left(E_{x} / E_{y}\right) \leq 90^{\circ}
\end{aligned}
$$

Ambiguities arising from the inversion of trigonometric functions are settled if the upper and lower limits of all quantities are considered.

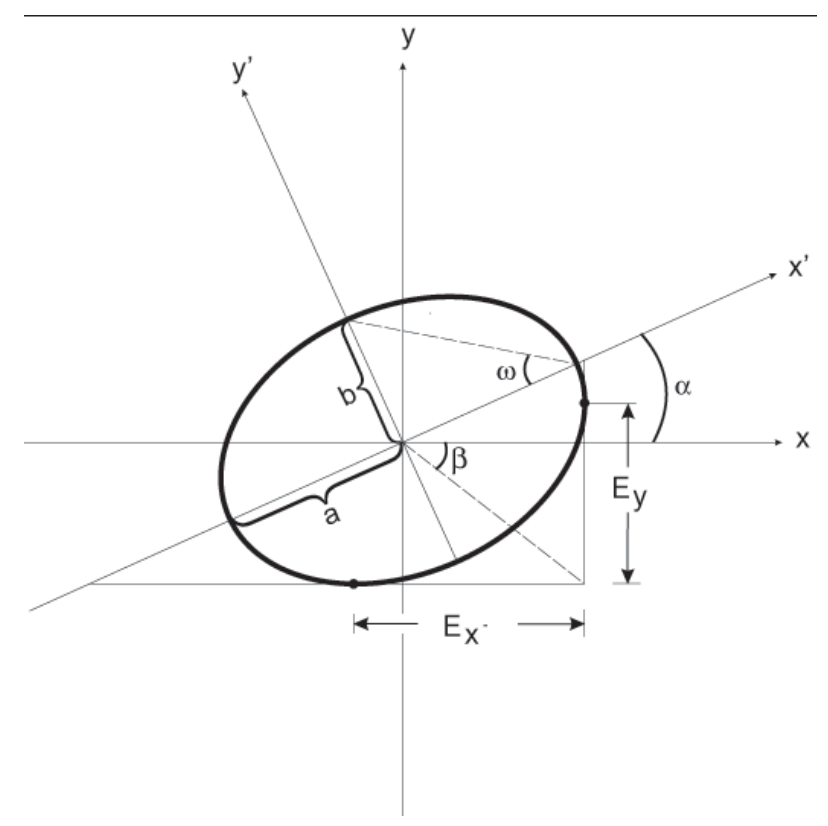

Figure 2: Elliptically polarized light defined by the azimuth $\alpha$ and the ellipticity $\omega$ 


\section{Basic equation of ellipsometry}

A typical ellipsometric experiment is depicted in Fig. 3. Light with a well defined state of polarization is incident on a sample. The reflected light usually differs in its state of polarization and these changes are measured and quantified in an ellipsometric experiment.

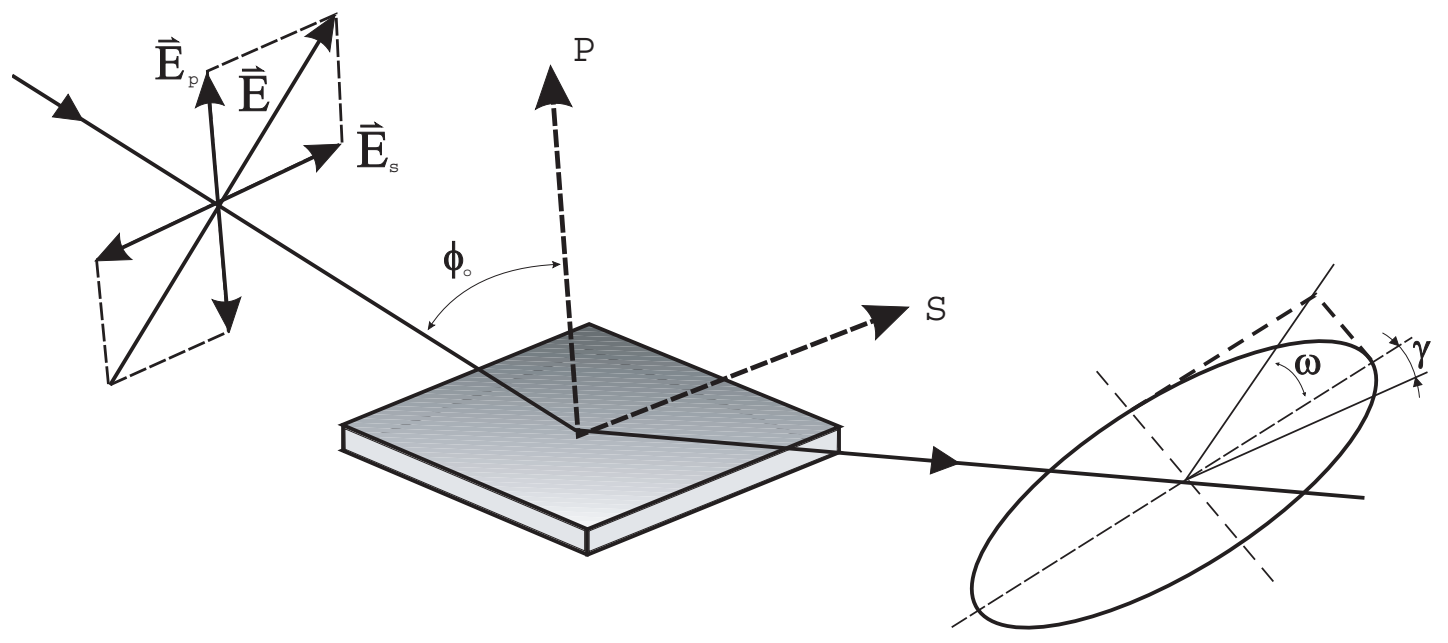

Figure 3: Ellipsometric experiment in reflection mode

The mathematical description is best done within the laboratory frame of reference defined by the plane of incidence. The propagation direction of the beam and the normal of the reflecting surface define the plane of incidence. Light with an electric field vector oscillating within the plane of incidence ( $\hat{p}$-light) remains linearly polarized upon reflection and the same holds for $\hat{s}$-light with $\vec{E}$ perpendicular to the plane of incidence. For this reason $\hat{p}$ and $\hat{s}$-light are also called Eigen-polarizations of isotropic media or uniaxial perpendicular media. This consideration makes it obvious that this frame of reference is distinct. Incident and reflected beam can be described by their corresponding Jones vector :

$$
\vec{E}_{\mathrm{inc}}=\left(\begin{array}{c}
\left|E_{\mathrm{p}}^{\mathrm{i}}\right| e^{i \delta_{\mathrm{p}}^{\mathrm{i}}} \\
\left|E_{\mathrm{s}}^{\mathrm{i}}\right| e^{i \delta_{\mathrm{s}}^{\mathrm{i}}}
\end{array}\right) \quad \vec{E}_{\mathrm{refl}}=\left(\begin{array}{c}
\left|E_{\mathrm{p}}^{\mathrm{r}}\right| e^{i \delta_{\mathrm{p}}^{\mathrm{r}}} \\
\left|E_{\mathrm{s}}^{\mathrm{r}}\right| e^{i \delta_{\mathrm{s}}^{\mathrm{r}}}
\end{array}\right)
$$

Two quantities $\Psi$ and $\Delta$ are introduced in order to describe the changes in the state of polarization.

$$
\Delta=\left(\delta_{\mathrm{p}}^{\mathrm{r}}-\delta_{\mathrm{s}}^{\mathrm{r}}\right)-\left(\delta_{\mathrm{p}}^{\mathrm{i}}-\delta_{\mathrm{s}}^{\mathrm{i}}\right)
$$




$$
\tan \Psi \frac{\left|E_{\mathrm{p}}^{\mathrm{r}}\right| /\left|E_{\mathrm{p}}^{\mathrm{i}}\right|}{=\overline{\overline{\mathrm{s}}}\left|/ E_{\mathrm{s}}^{\mathrm{r}}\right| / E_{\mathrm{s}}^{\mathrm{i}} \mid}
$$

Changes in the ratio of the amplitudes are described as the tangent of the angle $\Psi$. It will turn out later that $\Psi$ can be directly measured.

The reflectivity properties of a sample within a given experiment are given by the corresponding reflection coefficients $r_{p}$ and $r_{s}$. The reflection coefficient is a complex quantity that accounts for changes in phase and amplitude of the reflected electric field $E^{\mathrm{r}}$ with respect to the incident one $E^{\mathrm{i}}$.

$$
r_{\mathrm{p}}=\frac{\left|E_{\mathrm{p}}^{\mathrm{r}}\right|}{\left|E_{\mathrm{p}}^{i}\right|} e^{i\left(\delta_{\mathrm{p}}^{\mathrm{r}}-\delta_{\mathrm{p}}^{i}\right)} \quad r_{\mathrm{s}}=\frac{\left|E_{\mathrm{s}}^{\mathrm{r}}\right|}{\left|E_{\mathrm{s}}^{i}\right|} e^{i\left(\delta_{\mathrm{s}}^{\mathrm{r}}-\delta_{\mathrm{s}}^{i}\right)}
$$

Interference cannot be observed between orthogonal beams and hence $\hat{p}$ - and $\hat{s}$-light do not influence each other and can be separately treated. With these definitions the basic equation of ellipsometry is obtained

$$
\tan \Psi \cdot e^{i \Delta}=\frac{r_{\mathrm{p}}}{r_{\mathrm{s}}}=\rho=\Re(\rho)+i \Im(\rho)
$$

Eqn. (11) relates the quantities $\Psi$ and $\Delta$ with the reflectivity properties of the sample. The following two section discuss various ways to measure $\Delta$ and $\Psi$ and the theory and algorithm used for a calculation of the complex reflectivity coefficient. Some ellipsometers are designed in a way that they measure directly the real and imaginary part of the complex quantity $\rho$ instead of $\Psi$ and $\Delta$. Eqn. (11) allows a conversion between both notations.

\section{Design of an ellipsometer}

Many different designs of ellipsometers have been suggested and a good overview is presented in Azzam and Bashara [3]. Here we discuss common roots of all arrangements and the underlying theory.

The layout of a typical ellipsometer is depicted in Fig. 4. The main components are a polarizer $\mathrm{P}$ which produces linearly polarized light, a compensator $\mathrm{C}$ which introduces a defined phase retardation of one field component with respect to the orthogonal one, the sample $S$, the analyzer $A$ and a detector. 


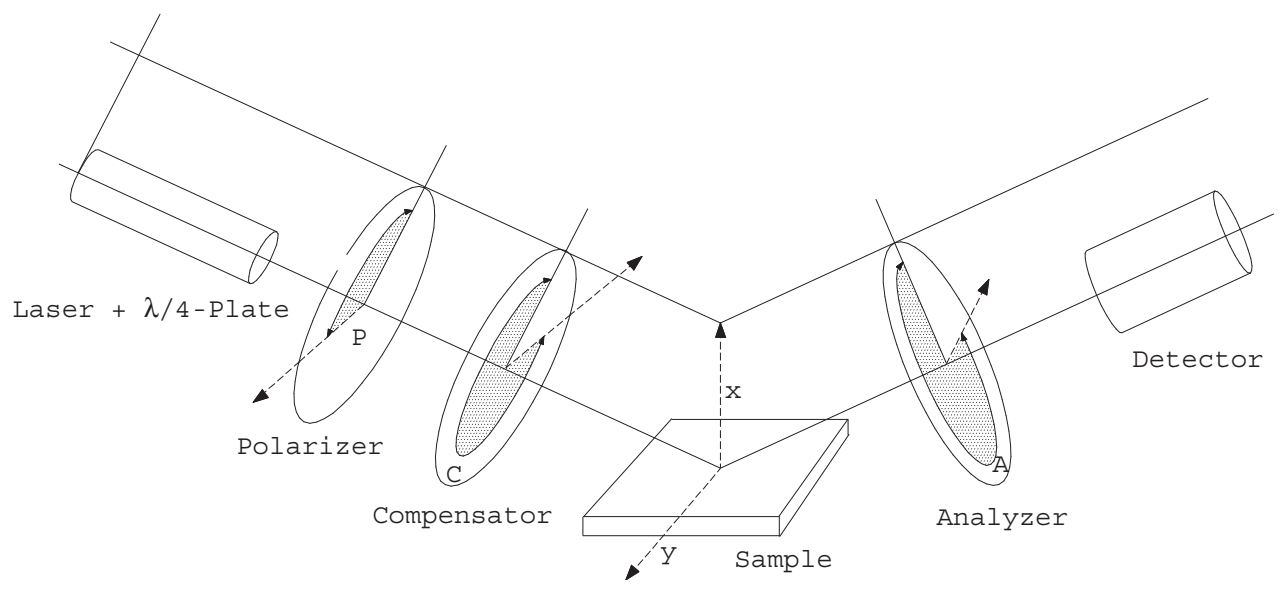

Figure 4: Ellipsometer in a PCSA-configuration

This setup allows the determination of the unknown ellipsometric angles and can be operated in various modes. Each optical component modifies the state of polarization. Since any state of polarization can be represented by a complex Jones vector consisting of two columns, the effect of each optical components is described by a complex $2 \times 2$ matrix. The Jones formalism provides an elegant means for a quantitative description [4].

$$
\left(\begin{array}{c}
E_{x} \\
E_{y}
\end{array}\right)^{J a}=\left(\begin{array}{cc}
T_{11} & T_{12} \\
T_{21} & T_{22}
\end{array}\right)^{J}\left(\begin{array}{c}
E_{x} \\
E_{y}
\end{array}\right)^{\mathrm{Je}}=\mathbf{T}^{J} \vec{E}^{J e}
$$

The superscript $J$ refers to the optical component $J \in[\mathrm{PCSA}]$; the subscripts $e$ and $a$ refer to the $\vec{E}$-vector before and after the component. Each of the optical components including the sample possesses a distinct coordinate system in which the corresponding matrix is diagonal. For example a compensator consist of a birefringent material cut to a thin plate of a defined thickness $t$. Optically it possesses a fast and a slow axis. Linearly polarized light oscillating parallel to either axis remains linearly polarized, however, since the corresponding refractive indices of fast $n_{\mathrm{f}}$ and slow axis $n_{\mathrm{s}}$ differ, they travel with different speed which leads to a phase shift $\phi=\left(n_{\mathrm{f}}-n_{\mathrm{s}}\right) \cdot t \cdot 2 \pi / \lambda$ between the two components. Similar expressions can be given for each component: 
component coordinate system

Polarizer

$t_{P}=$ transmission axis

$e_{P}=$ extinction axis

Compensator

$s_{c}=$ slow axis

$l_{c}=$ fast axis

with $\quad \rho_{c}=t_{c} e^{i \delta_{c}}=\frac{\left|E_{s}^{C a}\right|}{\left|E_{l}^{C a}\right|} e^{i\left(\delta_{s}-\delta_{l}\right)}$

eigenpolarization

Sample

$p$ parallel to plane of incidence

$s$ perpendicular to plane of incidence

Analyzer

\section{Jones-Matrix}

$$
\mathbf{T}^{P}=\left(\begin{array}{ll}
1 & 0 \\
0 & 0
\end{array}\right)
$$

$\mathbf{T}^{C}=\left(\begin{array}{ll}1 & 0 \\ 0 & \rho_{c}\end{array}\right)$

$\mathbf{T}^{S}=\left(\begin{array}{cc}r_{\mathrm{p}} & 0 \\ 0 & r_{\mathrm{s}}\end{array}\right)$

$\mathbf{T}^{A}=\left(\begin{array}{ll}1 & 0 \\ 0 & 0\end{array}\right)$

The simple diagonal matrix is only valid in the coordinate system of the component. A matrix $\mathbf{R}$ is required to transform the vector between the coordinate systems of adjacent components.

$$
\vec{E}_{x y}^{J+1, e}=\mathbf{R}(\alpha) \vec{E}_{x y}^{J, a} \quad \text { with } \quad \mathbf{R}(\alpha)=\left(\begin{array}{cc}
\cos \alpha & \sin \alpha \\
-\sin \alpha & \cos \alpha
\end{array}\right)
$$

The setting of the optical components is defined by the angles $P, A$ and $C$ of its distinct axis, with respect to the plane of incidence. An angle $C=-45^{\circ}$ means the fast axis of the compensator is set to an angle of $-45^{\circ}$ with respect to the plane of incidence.

With these tools we can describe the $E$-vector at the detector as a function of the setting 
of all components including the unknown reflectivity properties of the sample.

$$
\vec{E}_{e_{A} t_{A}}^{A, a}=\mathbf{T}^{A} \mathbf{R}(A) \mathbf{T}^{S} \mathbf{R}(-C) \mathbf{T}^{C} \mathbf{R}(C-P) \vec{E}_{e_{P} t_{P}}^{P, a}
$$

To understand Equation (14), one must first realize that the multiplication always goes from right to left. Hence, the above mathematical formula can be described as follows: linear polarized light exits the polarizer in the polarizer's frame of reference, then is rotated to the coordinate system of the compensator by the matrix operator $R(C-P)$, then the compensator acts on the state of polarization as given by $T_{C}$, then the exiting light from the compensator is rotated to the coordinate system of the sample by the matrix operator $R(-C)$ an so on. The multiplication given by Equation (14) yields:

$$
\begin{aligned}
\vec{E}_{e_{A} t_{A}}^{A, a} & =\left(\begin{array}{c}
E_{t_{A}}^{A, a} \\
0
\end{array}\right)=\left(\begin{array}{l}
1 \\
0
\end{array}\right) \gamma E_{t_{P}}^{P, a}\left\{\Omega_{1}+\Omega_{2}\right\} \\
\Omega_{1} & =R_{\mathrm{p}} \cos A\left[\cos C \cos (C-P)-\rho_{c} \sin C \sin [(C-P)]\right. \\
\Omega_{2} & =R_{\mathrm{s}} \sin A\left[\sin C \cos (C-P)+\rho_{c} \cos C \sin [(C-P)]\right.
\end{aligned}
$$

$\gamma$ accounts for the attenuation of the light intensity. Additional components in the optical path, for example the cell windows, should not change the state of polarization and can therefore be neglected. The intensity at the detector is proportional to

$$
I \propto\left|\vec{E}_{e_{A} t_{A}}^{A, a}\right|^{2}
$$

The Jones matrix algorithm leads to the desired relation between the intensity at the detector and the setting of all optical components. The unknown reflectivity coefficients can be retrieved in various manners and the applied measurement scheme names the method. Rotating analyzer means recording the intensity as a function of the setting of the analyzer and work out the unknown ellipsometric angles by a Fourier analysis. Polarization modulation ellipsometry uses a variable phase retardation $\delta_{c}$ for a calculation of the ellipsometric angles. Polarization modulation uses an electro-optic or acusto-optic modulator driven at a high frequency. The measurement is fast, however, there are also some inherent problems due to an undesired interferometric contribution of the modulator to the signal which cannot be separated from the contribution of the sample. The technique is very well suited to follow relative changes. 
A particular successful implementation is Nullellipsometry which eliminates many intrinsic errors due to slight misalignments of the sample. Within Nullellipsometry the setting of the optical components is chosen such that the light at the detector vanishes. This requires that the angle dependent term $\left\{\Omega_{1}+\Omega_{2}\right\}$ of eqn. (15) vanishes. A given elliptical state of polarization of the incident light leads to linear polarized light after reflection and can be completely extinguished with an analyzer.

$$
\frac{r_{\mathrm{p}}}{r_{\mathrm{s}}}=-\tan A \frac{\tan C+\rho_{c} \tan (C-P)}{1-\rho_{c} \tan C \tan (C-P)} \quad \text { for } \quad I=0
$$

This equation can be further simplified by using a high precision quarter waveplate as a compensator $\left(t_{C}=1, \delta_{C}=\pi / 2 \Longrightarrow \rho_{c}=-i\right)$ fixed to $C= \pm 45^{\circ}$. With eqn. (11) a further simplification of the eqn. (17) can be achieved.

$$
\begin{array}{ll}
\tan \Psi e^{i \Delta}=\frac{r_{\mathrm{p}}}{r_{\mathrm{s}}}=\tan A_{0} \exp \left[i\left(2 P_{0}+\frac{\pi}{2}\right)\right] & \text { if } \quad C=-45^{\circ} \\
\tan \Psi e^{i \Delta}=\frac{r_{\mathrm{p}}}{r_{\mathrm{s}}}=-\tan A_{0} \exp \left[i\left(\frac{\pi}{2}-2 P_{0}\right)\right] & \text { if } \quad C=45^{\circ}
\end{array}
$$

Eqn. (18) links the quantities $\Delta$ an $\Psi$ to the null settings of the polarize $P_{0}$ and analyzer $A_{0}$. Once a setting $\left(P_{0}, A_{0}\right)$ has been determined which provides a complete cancellation of the light, then the same holds for the pair $\left(\tilde{P}_{0}, \tilde{A}_{0}\right)$.

$$
\left(\tilde{P}_{0}, \tilde{A}_{0}\right)=\left(P_{0}+90^{\circ}, 180^{\circ}-A_{0}\right) \quad \text { if } I=0 \text { for }\left(P_{0}, A_{0}\right)
$$

These nontrivial pairs of nullsettings are refered as ellipsometric zones. Measurements in various zones lead to a high accuracy in the determination of absolute values. Many intrinsic small errors due to misalignment are cancelled by this scheme.

So far we introduced two quantitites which account for changes in the state of reflection upon reflection. We also discussed how these quantities can be measured. The next chapter deals with the theory of reflection and illustrates means for a calculation of the ellipsometric angles of a given optical layer system.

\section{Theory of reflection}

In the following section a procedure for a calculation of the reflectivity coefficients $r_{p}$ and $r_{s}$ (and consequently the ellipsometric angles) first published by Lekner [6] will be described. 
This method is certainly not the easiest one for the calculation of the reflectivity properties of a single homogenous layer at the interface between two bulk phases, but is advantageous, if the layer has an inner structure, e.g. a continuously varying refractive index normal to the interfaces. For just a few refractive index profiles it is possible to calculate $r_{p}$ and $r_{s}$ exactly, but in most cases approximations must be used: A continuously varying refractive index profile is subdivided into many thin layers. This method uses matrices to relate the electrical field and its derivative in between adjacent layer and matrices to account for changes of the phase in each layer. In every layer the refractive index is assumed to be constant. Obviously this approximation gets the better the finer the subdivision is.

For the derivation we use a rectangular coordinate system with the $\hat{z}$-axis normal to the interfaces pointing from the incident medium to the substrate. This means that the interfaces of the layer structure are planes of constant $z_{n}$. The $\hat{x}, \hat{z}$-plane is the plane of incidence.

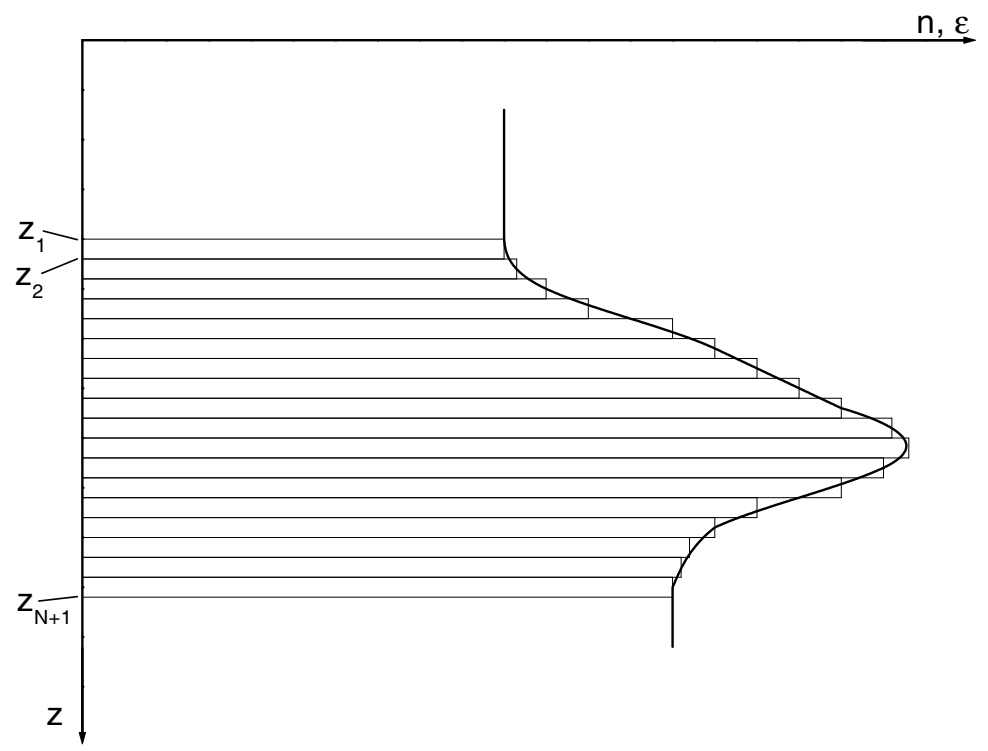

Figure 5: Subdivision of a refractive index profile into step profiles

We start with Maxwell's equation. In the case of no magnetization, no free charges, no current in and linear polarizability of the sample they read:

$$
\nabla \times \vec{B}=\frac{\epsilon}{c^{2}} \cdot \frac{\partial \vec{E}}{\partial t}
$$




$$
\begin{aligned}
& \nabla \times \vec{E}=-\frac{\partial \vec{B}}{\partial t} \\
& \nabla \cdot \vec{B}=0 \\
& \nabla \cdot \vec{E}=0
\end{aligned}
$$

with $\vec{B}$ representing the magnetic field, $\vec{E}$ the electric field, $\epsilon(z)=n(z)^{2}$ the dielectric function and $c$ the velocity of light in vacuo.

For the $\vec{B}$ - and the $\vec{E}$-field a plane wave ansatz is used:

$$
\vec{B}=\vec{B}_{0} \cdot e^{i(\vec{k} \cdot \vec{r}-\omega t)}, \quad \vec{E}=\vec{E}_{0} \cdot e^{i(\vec{k} \cdot \vec{r}-\omega t)}
$$

This ansatz fulfills Maxwell's equations, if the following relationship between the wave vector $\vec{k}$ and the angular frequency $\omega$ of the propagating wave is obeyed:

$$
\vec{k}^{2}+\epsilon \frac{\omega^{2}}{c^{2}}=0
$$

and if additionally $\vec{B}_{0}, \vec{E}_{0}$ and $\vec{k}$ are normal to each other. Thus eqn. (20) and eqn. (21) can be further simplified:

$$
\nabla \times \vec{B}=-i \frac{\epsilon \omega}{c^{2}} \vec{E} \quad \nabla \times \vec{E}=i \omega \vec{B}
$$

Let's consider $\hat{s}$-polarized light, that can be represented in the following way in the coordinate system:

$$
\vec{E}_{\mathrm{s}}=\left(\begin{array}{c}
0 \\
E_{y} \\
0
\end{array}\right)
$$

Inserting $\vec{E}_{\mathrm{s}}$ in eqn. (26) results in three equations for $E_{y}$ :

$$
-\frac{\partial E_{y}}{\partial z}=i \frac{\omega}{c} B_{x} \quad \frac{\partial E_{y}}{\partial x}=i \frac{\omega}{c} B_{z} \quad \frac{\partial B_{x}}{\partial z}-\frac{\partial B_{z}}{\partial x}=-i \frac{\omega}{c} E_{y}
$$

After elimination of $B_{x}$ and $B_{z}$ one obtains a differential equation in $E_{y}$ which can be separated:

$$
\frac{\partial^{2} E_{y}}{\partial x^{2}}+\frac{\partial^{2} E_{y}}{\partial z^{2}}+\epsilon \frac{\omega^{2}}{c^{2}} E_{y}=0
$$

The ansatz $E_{y}(x, z, t)=e^{i\left(k_{x} x-\omega t\right)} \cdot E(z)$ leads to a differential equation in $E(z)$ :

$$
\frac{d^{2} E(z)}{d z^{2}}+q^{2} E(z)=0,
$$


with $q$ being the $\hat{z}$-component of the wave vector:

$$
q^{2}=\epsilon \frac{\omega^{2}}{c^{2}}-k_{x}^{2}=k^{2}-k_{x}^{2} \quad \text { or } \quad q=\sqrt{\epsilon} \frac{\omega}{c} \cdot \cos \varphi
$$

and $\varphi$ representing the angle of incidence (defined as the angle between the surface normal and the direction of propagation of the light beam). Since $E(z)$ is continuous at every interface, it is obvious from eqn. (30), that also $d E(z) / d z$ is continuous, if $d \epsilon \neq \infty$.

Eqn. (30) can thus be split into two coupled differential equations of first order:

$$
\frac{d E(z)}{d z}=D(z) \quad \text { und } \quad \frac{d D(z)}{d z}=-q^{2} E
$$

If $q$ takes the value $q_{n}$ within a layer located between $z_{n}$ and $z_{n+1}$, and $E_{n}$ and $D_{n}$ are the values of the electric field and its derivative at $z_{n}$, the solution of eqn. (32) in $z_{n} \leq z \leq z_{n+1}$ is:

$$
\begin{aligned}
& E(z)=E_{n} \cos q_{n}\left(z-z_{n}\right)+\frac{D_{n}}{q_{n}} \sin q_{n}\left(z-z_{n}\right), \\
& D(z)=D_{n} \cos q_{n}\left(z-z_{n}\right)-E_{n} q_{n} \sin q_{n}\left(z-z_{n}\right) .
\end{aligned}
$$

Since $E$ and $D$ are continuous at the interfaces, it follows that

$$
\begin{aligned}
& E_{n+1}=E_{n} \cos \delta_{n}+\frac{D_{n}}{q_{n}} \sin \delta_{n} \\
& D_{n+1}=D_{n} \cos \delta_{n}-E_{n} q_{n} \sin \delta_{n},
\end{aligned}
$$

with

$$
\delta_{n}=q_{n}\left(z_{n+1}-z_{n}\right)
$$

representing the phase shift encountered while propagating through the layer. The eqn. (35) can be expressed in a matrix form:

$$
\left(\begin{array}{c}
E_{n+1} \\
D_{n+1}
\end{array}\right)=\left(\begin{array}{cc}
\cos \delta_{n} & \frac{\sin \delta_{n}}{q_{n}} \\
-q_{n} \sin \delta_{n} & \cos \delta_{n}
\end{array}\right)\left(\begin{array}{c}
E_{n} \\
D_{n}
\end{array}\right)=\mathbf{M}_{n s} \cdot\left(\begin{array}{c}
E_{n} \\
D_{n}
\end{array}\right)
$$

where $\mathbf{M}_{n s}$ describes the influence of the $n$-th layer on the $\hat{s}$-polarized wave.

The matrices for $\hat{p}$-polarized light can be calculated analogously. Since $\hat{p}$-light has nonvanishing $\hat{x}$ - and $\hat{z}$-components of the electrical field in the chosen coordinate system, 
it is more convenient to use the linearly coupled magnetic field $\vec{B}$, which has just one nonvanishing component, instead of $\vec{E}$.

$$
\vec{B}=\left(\begin{array}{c}
0 \\
B_{y} \\
0
\end{array}\right)=\left(\begin{array}{c}
0 \\
e^{i\left(k_{x} x-\omega t\right)} B(z) \\
0
\end{array}\right)
$$

Insertion of $\vec{B}$ into Maxwell's equations and simplification lead to a differential equation in $B(z)$ :

$$
\frac{d}{d z}\left(\frac{1}{\epsilon} \frac{d B(z)}{d z}\right)+\frac{q^{2}}{\epsilon} B(z)=0
$$

that can be split into two equations of first order again:

$$
\frac{1}{\epsilon} \frac{d B(z)}{d z}=C \quad \text { and } \frac{d C(z)}{d z}=-\frac{q^{2}}{\epsilon} B(z)
$$

Their solutions within a layer $n$ resemble those of the $\hat{s}$-polarized light (eqn. (33) and eqn. (34)):

$$
\begin{aligned}
& B(z)=B_{n} \cos q_{n}\left(z-z_{n}\right)+\frac{\epsilon_{n}}{q_{n}} C_{n} \sin q_{n}\left(z-z_{n}\right) \\
& C(z)=C_{n} \cos q_{n}\left(z-z_{n}\right)-\frac{q_{n}}{\epsilon_{n}} B_{n} \sin q_{n}\left(z-z_{n}\right)
\end{aligned}
$$

It follows from eqn. (20) and eqn. (39) that $B(z)$ and $C(z)$ are continuous at the layers' interfaces, if $d \epsilon \neq \infty$. This can be used again to relate the values of $B$ and $\mathrm{C}$ at neighboring interfaces.

$$
\left(\begin{array}{c}
B_{n+1} \\
C_{n+1}
\end{array}\right)=\left(\begin{array}{cc}
\cos \delta_{n} & \frac{\epsilon_{n}}{q_{n}} \sin \delta_{n} \\
-\frac{q_{n}}{\epsilon_{n}} \sin \delta_{n} & \cos \delta_{n}
\end{array}\right)\left(\begin{array}{c}
B_{n} \\
C_{n}
\end{array}\right)=\mathbf{M}_{n p}\left(\begin{array}{c}
B_{n} \\
C_{n}
\end{array}\right)
$$

Now it is clear how to calculate the reflectivity coefficients of such a layer structure: The layers' matrices $\mathbf{M}_{n}$ have to be multiplied consecutively, separately for $\hat{p}$ - and $\hat{s}$-light:

$$
\mathbf{M}=\left(\begin{array}{ll}
m_{11} & m_{12} \\
m_{21} & m_{22}
\end{array}\right)=\mathbf{M}_{N} \cdot \mathbf{M}_{N-1} \cdots \mathbf{M}_{2} \cdot \mathbf{M}_{1}
$$

The resulting matrices $\mathbf{M}_{s}$ and $\mathbf{M}_{p}$ relate the fields before and after the layer structure. 
Before it $(f)$ there are incident and reflected wave, behind it $(h)$ just the transmitted one:

$$
\begin{aligned}
& E_{1}=e^{i q_{f} z_{1}}+r_{s} e^{-i q_{f} z_{1}} \Longrightarrow \quad D_{1}=i q_{f}\left(e^{i q_{f} z_{1}}-r_{s} e^{-i q_{f} z_{1}}\right) \\
& E_{N+1}=t_{s} e^{i q_{h} z_{N+1}} \quad \Longrightarrow D_{N+1}=i q_{h} t_{s} e^{i q_{h} z_{N+1}} \\
& B_{1}=e^{i q_{f} z_{1}}-r_{p} e^{-i q_{f} z_{1}} \Longrightarrow \quad C_{1}=i q_{f}\left(e^{i q_{f} z_{1}}+r_{p} e^{-i q_{f} z_{1}}\right) \\
& B_{N+1}=\sqrt{\frac{\epsilon_{h}}{\epsilon_{f}}} t_{p} e^{i q_{h} z_{N+1}} \Longrightarrow C_{N+1}=i q_{h} \sqrt{\frac{\epsilon_{h}}{\epsilon_{f}}} t_{p} e^{i q_{h} z_{N+1}}
\end{aligned}
$$

Thus the following relations evolve:

$$
\begin{array}{r}
\left(\begin{array}{c}
t_{s} e^{i q_{h} z_{N+1}} \\
i q_{h} t_{s} e^{i q_{h} z_{N+1}}
\end{array}\right)=\left(\begin{array}{ll}
m_{11 s} & m_{12 s} \\
m_{21 s} & m_{22 s}
\end{array}\right)\left(\begin{array}{c}
e^{i q_{f} z_{1}}+r_{s} e^{-i q_{f} z_{1}} \\
i q_{f}\left(e^{i q_{f} z_{1}}-r_{s} e^{-i q_{f} z_{1}}\right)
\end{array}\right) \\
\left(\begin{array}{c}
\sqrt{\frac{\epsilon_{h}}{\epsilon_{f}}} t_{p} e^{i q_{h} z_{N+1}} \\
i q_{h} \sqrt{\frac{\epsilon_{h}}{\epsilon_{f}}} t_{p} e^{i q_{h} z_{N+1}}
\end{array}\right)=\left(\begin{array}{ll}
m_{11 p} & m_{12 p} \\
m_{21 p} & m_{22 p}
\end{array}\right)\left(\begin{array}{c}
e^{i q_{f} z_{1}}-r_{p} e^{-i q_{f} z_{1}} \\
i q_{f}\left(e^{i q_{f} z_{1}}+r_{p} e^{-i q_{f} z_{1}}\right)
\end{array}\right)
\end{array}
$$

These equations can be solved for $r_{s}$ and $r_{p}$, resulting in two formally identical expressions for the two coefficients:

$$
\begin{array}{r}
r_{s}=e^{2 i q_{f} z_{1}} \frac{q_{f} q_{h} m_{12 s}+m_{21 s}-i q_{h} m_{11 s}+i q_{f} m_{22 s}}{q_{f} q_{h} m_{12 s}-m_{21 s}+i q_{h} m_{11 s}+i q_{f} m_{22 s}} \\
-r_{p}=e^{2 i q_{f} z_{1}} \frac{\frac{q_{f} q_{h}}{\epsilon_{f} \epsilon_{h}} m_{12 p}+m_{21 p}-i \frac{q_{h}}{\epsilon_{h}} m_{11 p}+i \frac{q_{f}}{\epsilon_{f}} m_{22 p}}{\frac{q_{f} q_{h}}{\epsilon_{f} \epsilon_{h}} m_{12 p}-m_{21 p}+i \frac{q_{h}}{\epsilon_{h}} m_{11 p}+i \frac{q_{f}}{\epsilon_{f}} m_{22 p}}
\end{array}
$$

Up to now the only simplification is the discretization of the continuous refractive index profile. The next step is a reduction of the computational effort by using a Taylorapproximation up to the second order in the phaseshift $\delta_{n}$. This approximation is valid if the discrete layer thicknesses are much smaller than the wavelength of the light: in other words a sufficiently high number of layers is required.

$$
\mathbf{M}_{n}=\left(\begin{array}{cc}
\cos \delta_{n} & \frac{\sin \delta_{n}}{q_{n}} \\
-q_{n} \sin \delta_{n} & \cos \delta_{n}
\end{array}\right) \quad \text { and } \quad\left(\begin{array}{cc}
\cos \delta_{n} & \frac{\epsilon_{n}}{q_{n}} \sin \delta_{n} \\
-\frac{q_{n}}{\epsilon_{n}} \sin \delta_{n} & \cos \delta_{n}
\end{array}\right)
$$

for $\hat{s}^{-}$and $\hat{p}$-polarized light simplify to

$$
\mathbf{M}_{n} \approx\left(\begin{array}{cc}
1-\frac{\delta_{n}^{2}}{2} & \frac{\delta_{n}}{q_{n}} \\
-q_{n} \delta_{n} & 1-\frac{\delta_{n}^{2}}{2}
\end{array}\right) \quad \text { and } \quad\left(\begin{array}{cc}
1-\frac{\delta_{n}^{2}}{2} & \frac{\epsilon_{n} \delta_{n}}{q_{n}} \\
-\frac{q_{n} \delta_{n}}{\epsilon_{n}} & 1-\frac{\delta_{n}^{2}}{2}
\end{array}\right)
$$

With just a slight increase of computational effort the approximation can be greatly improved by using layers with linearly varying dielectric functions $\epsilon$ as depicted in fig.6 instead of those with constant $\epsilon$. 


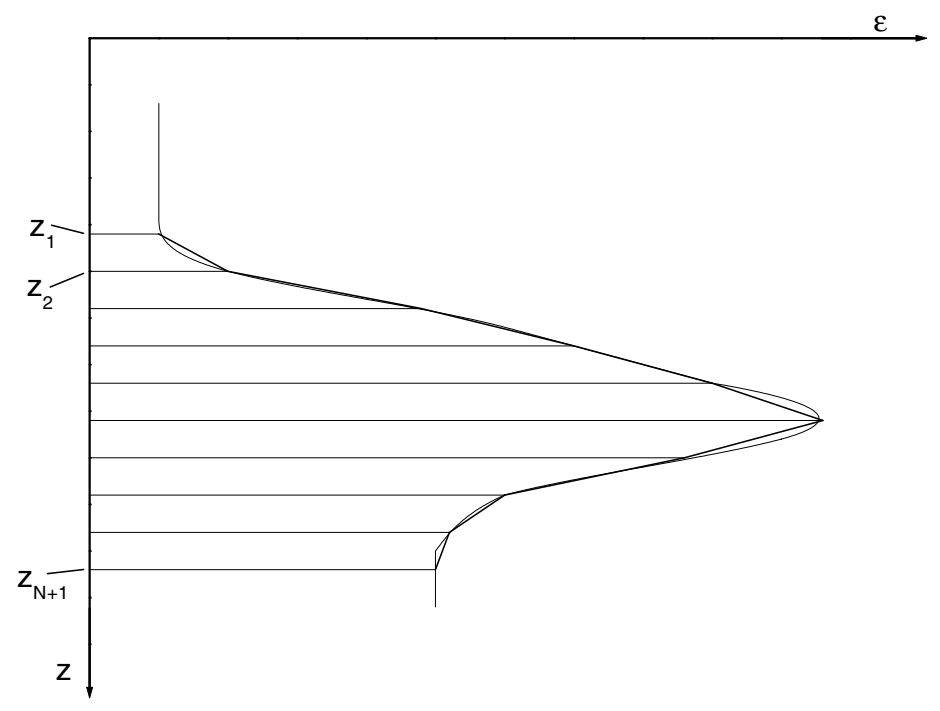

Figure 6: Approximation of the real dielectric function by a system of layers with linearly varying dielectric function $\epsilon$

Using this ansatz the components of the individual layer matrices adopt the following form:

$$
\begin{aligned}
& m_{11 s}=1+\left(z_{n+1}-z_{n}\right)^{2}\left(\frac{k_{x}^{2}}{2}-\frac{\omega^{2}}{c^{2}} \frac{2 \epsilon_{n}+\epsilon_{n+1}}{6}\right) \\
& m_{12 s}=z_{n+1}-z_{n} \\
& m_{21 s}=\left(z_{n+1}-z_{n}\right)\left(k_{x}^{2}-\frac{\omega^{2}}{c^{2}} \frac{\epsilon_{n}+\epsilon_{n+1}}{2}\right) \\
& m_{22 s}=1+\left(z_{n+1}-z_{n}\right)^{2}\left(\frac{k_{x}^{2}}{2}-\frac{\omega^{2}}{c^{2}} \frac{\epsilon_{n}+2 \epsilon_{n+1}}{6}\right) \\
& m_{11 p}=1+\left(z_{n+1}-z_{n}\right)^{2}\left(k_{x}^{2} \frac{2 \epsilon_{n}+\epsilon_{n+1}}{6 \epsilon_{n}}-\frac{\omega^{2}}{c^{2}} \frac{\epsilon_{n}+2 \epsilon_{n+1}}{6}\right) \\
& m_{12 p}=\left(z_{n+1}-z_{n}\right) \frac{\epsilon_{n}+\epsilon_{n+1}}{2} \\
& m_{21 p}=\left(z_{n+1}-z_{n}\right)\left(\frac{k_{x}^{2}}{2}\left(\frac{1}{\epsilon_{n}}+\frac{1}{\epsilon_{n+1}}\right)-\frac{\omega^{2}}{c^{2}}\right) \\
& m_{22 p}=1+\left(z_{n+1}-z_{n}\right)^{2}\left(k_{x}^{2} \frac{\epsilon_{n}+2 \epsilon_{n+1}}{6 \epsilon_{n+1}}-\frac{\omega^{2}}{c^{2}} \frac{2 \epsilon_{n}+\epsilon_{n+1}}{6}\right)
\end{aligned}
$$

Setting $\epsilon_{n}=\epsilon_{n+1}$ within a layer in eqn. (52)yields the step profile approximation eqn. (51). $>$ From the equations for $r_{p}$ and $r_{s}$, eqn. (49) and eqn. (48), the ellipsometric angles $\Delta$ and $\Psi$ of arbitrary layer structures can be calculated using the basic equation of ellipsometry eqn. 11 . 


\section{Ellipsometry applied to ultrathin films}

The previous section described an algorithm for the calculation on the reflectivity coefficients of given refractive index profiles. Profiles are only of importance if their characteristic length scale is comparable to that of the probing beam. In many cases, i.e. adsorption layer of nonionic surfactants at the air-water interface, there is a striking mismatch between interfacial height $h$ and the wavelength of light $\lambda$. As a result certain peculiarities exist which are discussed in this section [8].

The most striking limitation is a reduction of the measurable quantities. The presence of an organic monolayer (refractive index 1.3-1.6) with a thickness below $2.5 \mathrm{~nm}$ does not change the reflectivity $\left|r_{i}\right|^{2}$ and as a consequence there are no detectable changes in $\Psi$. In the thin film limith $\ll \lambda$ the data analysis relies only on a single parameter, namely changes in the phase $\Delta$. Unfortunately the number of independent data cannot be increased. Neither spectroscopic ellipsometry nor a variation of the angle of incidence yield new independent data, instead all quantities remain strongly coupled. A sound treatment is given in [9]. However, the sensitivity of an ellipsometric measurement can be significantly increased by the choice of the angle of incidence. A sensitivity analysis is given in [10].

The exact formula relating the reflectivity coefficients of a single homogeneous layer with refractive index $n_{1}=\sqrt{\epsilon_{1}}$ in between two infinite media $\left(n_{0}=\sqrt{\epsilon_{0}}\right.$ and $\left.n_{2}=\sqrt{\epsilon_{2}}\right)$ at an angle of incidence $\varphi$ is given by :

$$
\Delta=\arctan \frac{\operatorname{Im}\left(\frac{r_{p}}{r_{s}}\right)}{\operatorname{Re}\left(\frac{r_{p}}{r_{s}}\right)} \quad \text { with } \quad \begin{aligned}
r_{p} & =\left|r_{p}\right| \cdot e^{i \delta_{r, p}}=\frac{r_{0,1, p}+r_{1,2, p} e^{-i 2 \beta}}{1+r_{0,1, p} r_{1,2, p} e^{-i 2 \beta}} \\
r_{s} & =\left|r_{s}\right| \cdot e^{i \delta_{r, s}}=\frac{r_{0,1, s}+r_{1,2, s} e^{-i 2 \beta}}{1+r_{0,1, s} r_{1,2, s} e^{-i 2 \beta}}
\end{aligned}
$$

where the reflectivity coefficients $r_{0,1, p}, r_{1,2, p}, r_{0,1, s}$ and $r_{1,2, s}$ describing the reflection at refractive index jumps $n_{0} \rightarrow n_{1}$ and $n_{1} \rightarrow n_{2}$ for $\hat{p}$ - and $\hat{s}$-light are given by Fresnel's laws. $\beta=2 \pi \frac{h}{\lambda} \sqrt{n_{1}^{2}-n_{0}^{2} \sin ^{2} \varphi}$ accounts for the phase shift occuring in a single pass within the adsorption layer.

If the layer thickness $h$ is much smaller than the wavelength $\lambda$ of light it is justified to expand the complex reflectivity coefficients in a power series in terms of $h / \lambda$. The first 
term in this expansion describes reflection at a monolayer.

$$
\Delta \approx \frac{4 \sqrt{\epsilon_{0}} \epsilon_{2} \pi \cos \varphi \sin ^{2} \varphi}{\left(\epsilon_{0}-\epsilon_{2}\right)\left(\left(\epsilon_{0}+\epsilon_{2}\right) \cos ^{2} \varphi-\epsilon_{0}\right)} \cdot \frac{\left(\epsilon_{1}-\epsilon_{0}\right)\left(\epsilon_{2}-\epsilon_{1}\right)}{\epsilon_{1}} \cdot \frac{h}{\lambda}
$$

If the refractive index is varying over the height of the layer, the term

$$
\frac{\left(\epsilon_{1}-\epsilon_{0}\right)\left(\epsilon_{2}-\epsilon_{1}\right)}{\epsilon_{1}} \cdot h
$$

within eqn. (63) has to be replaced by an integral $\eta$ across the interface:

$$
\eta=\int \frac{\left(\epsilon-\epsilon_{0}\right)\left(\epsilon_{2}-\epsilon\right)}{\epsilon} d z
$$

An ellipsometric experiment on a monolayer yields a quantity proportional to $\eta$. A simplification of eqn. (55) reveals its physical meaning. Quite often, as for example in case of adsorption of organic compounds onto solid supports [7], $\epsilon_{2}$ exceeds $\epsilon$ of the monolayer while $\epsilon \approx \epsilon_{0}$.

Under these circumstances eqn. (55) can be further simplified:

$$
\eta=\frac{\epsilon_{2}-\epsilon_{0}}{\epsilon_{0}} \int\left(\epsilon-\epsilon_{0}\right) d z
$$

A linear relationship between $\epsilon$ and the prevailing concentration $c$ of amphiphile within the adsorption layer is well established [11]

$$
\epsilon=\epsilon_{0}+c \frac{\mathrm{d} \epsilon}{\mathrm{d} c}
$$

This relation yields a direct proportionality between the quantity $\eta$ and the adsorbed amount $\Gamma$.

$$
\eta=\frac{\epsilon_{2}-\epsilon_{0}}{\epsilon_{0}} \frac{\mathrm{d} \epsilon}{\mathrm{d} c} \int c d z=\frac{\epsilon_{2}-\epsilon_{0}}{\epsilon_{0}} \frac{\mathrm{d} \epsilon}{\mathrm{d} c} \Gamma
$$

None of the assumptions which lead to eqn. (58) apply for adsorption layers at the liquidair interface and hence eqn. (55) cannot be further simplified. The relationship between monolayer data and recorded changes remains obscure with no further simplifications possible on the basis of Maxwell's equations. A proportionality between $\Delta$ and $\Gamma$ may hold but it cannot be established within this theoretical framework. 


\section{Microscopic model for reflection}

Quantities such as refractive index or thickness are macroscopic quantities. Their meaning at sub-monolayer coverage is not an obvious one. To bridge these inherent difficulties several approaches have been developed aiming for a calculation of the optical properties of a monolayer based on microscopic quantitities [12]. We follow here mainly a model originally derived by Dignam et.al. [13, 14]. Explicit formulas are derived relating the changes in $\Delta$ to the polarizibility tensor of the adsorbed molecule and their number density at the interface.

The adsorption layer is treated as a two dimensional sheet of dipoles driven by the external laser field. The oscillating dipoles act as sources of radiation and the summation of all their contributions yields the reflected beam in the far field.

The adsorption layer is considered as homogeneous, fluctuations in the orientation or density occur on a length scale much smaller than the wavelength of light. These conditions are usually fulfilled for adsorption layers of soluble surfactants.

The decisive new quantitity introduced by Dignam et. al. is the surface susceptibility tensor $\vec{\gamma}$

$$
\vec{\gamma} E_{0}=4 \pi P t
$$

which links the external electric field $E_{0}$ to the resulting polarization per volume $\mathrm{P}, t$ represents the thickness of the adsorbed layer. The polarization is given by the vector sum of all dipole moments $\mu_{j}$ over all molecules $j$ :

$$
\vec{\gamma} E_{0}=4 \pi \sum_{j} \mu_{j}
$$

The local field at each dipole has two origins, the external laser field $E_{0}$ and the dipolar contribution which is proportional to $E_{0}$.

$$
E_{L}=E_{0}+\vec{\beta} E_{0}
$$

Each adsorbed molecule is characterized by its own tensor $\beta$. The induced dipole moment of a molecule with a polarizability $\alpha_{j}$ is given by

$$
\mu_{j}=\vec{\alpha}_{j} E_{j L}=\vec{\alpha}_{j}\left(1+\vec{\beta}_{j}\right) E_{0}
$$


with this notation the susceptibilty tensor $\vec{\gamma}$ reads:

$$
\vec{\gamma}=4 \pi \sum_{j} \vec{\alpha}_{j}\left(1+\vec{\beta}_{j}\right)
$$

In order to calculate $\beta$ it is desirable to introduce the field $E_{i j}$, which accounts for the electric field at the position $r_{i}$ generated by the dipole of molecule $j$.

$$
E_{i j}=\vec{f}_{i j} \mu_{j}
$$

$$
\begin{aligned}
& \vec{f}_{i j}=\left(3 \vec{\rho}_{i j}-1\right) / r_{i j}^{3} \quad \text { for } i \neq j \\
& \vec{f}_{i j}=0 \quad \text { for } i=j
\end{aligned}
$$

The projection operator $\vec{\rho}$ projects the induced dipole moment on the connection of the molecules at site $i$ and $j$.

$$
E_{i j}=\vec{f}_{i j} \vec{\alpha}_{i j}\left(1+\vec{\beta}_{j}\right) E_{0}
$$

With the relation:

$$
\sum_{j} E_{i j}=\vec{\beta}_{i} E_{o}
$$

$\vec{\beta}_{i}$ is given by

$$
\vec{\beta}_{i}=\sum_{j} \vec{f}_{i j} \vec{\alpha}_{i j}\left(1+\vec{\beta}_{j}\right)
$$

Each molecule is characterized by a tensor $\beta_{j}$. For a further calculation certain assumptions about the molecular distribution must be introduced. For adsorption layers of soluble surfactants at the air-water interface it is a good approximation to consider $\beta$ for all molecules equal. The underlying molecular pircture is is that the adsorption layer consists of one species of amphiphiles with a narrow angular distribution. 
Under these conditions:

$$
\beta=\sum_{j} \vec{f}_{i j} \vec{\alpha}_{j}(1+\beta)
$$

meaning that

$$
1+\beta=\left(1-\sum_{j} \vec{f}_{i j} \vec{\alpha}\right)^{-1}
$$

With equation 63 the surface susceptibility tensor reads:

$$
\vec{\gamma}=\frac{4 \pi N \vec{\alpha}}{\left(1-\sum_{j} \vec{f}_{i j}\right) \vec{\alpha}}
$$

The number of molecules per unit area is denoted by $N$. For an uniaxial film with its optical axis parallel to the surface normal the tangential $t$ and normal $n$ component of the susceptibility tensor read:

$$
\begin{aligned}
\gamma_{t} & =\frac{4 \pi N \alpha_{t}}{\left(1-4 \pi / 3\left(N / t_{e}\right) \alpha_{t}\right.} \\
\gamma_{n} & =\frac{4 \pi N \alpha_{n}}{\left(1-8 \pi / 3\left(N / t_{e}\right) \alpha_{n}\right.}
\end{aligned}
$$

The quantity $t_{e}$ can be regarded as an effective thickness as defined as

$$
t_{e}=\frac{8 / 3 \pi N}{\sum_{i \neq j} 1 / r_{i j}^{3}}
$$

If the adsorption layer can be mathematically represented as adsorbed molecules on a rectangular grid with an average next neighbour distance of $a$

$$
t_{e}=\frac{8 / 3 \pi a^{3} N}{\left(i^{2}+j^{2}\right)^{-3 / 2}}=0.935 a^{3} N
$$

At monolayer coverage $N=1 / a^{2}$ the effective thickness is $t_{e}=0.935 a$. The adsorption of molecules at the interface changes the optical properties of the system. The changes in the ellipsometric angles are given by 


$$
\ln \left(\frac{\tan \psi}{\tan \psi_{0}}\right)+i\left(\Delta-\Delta_{0}\right)=\delta L
$$

In the thin film limit only changes in $\Delta$ occur:

$$
\delta L_{v}=\frac{i 4 \pi}{\lambda} \cos \varphi \frac{\delta \gamma_{t}}{1-\epsilon_{2}}\left(1+\delta_{v}, p \frac{1-\epsilon_{s} \delta \gamma_{n} / \delta \gamma_{t}}{\cot ^{2} \varphi-1 / \epsilon_{2}}\right)
$$

$\delta \gamma_{n, t}$ denotes the difference of the susceptibility tensor between the film covered and the bare surface, $i=\sqrt{-1}, \varphi$ is the angle of incidence and $\lambda$ is the wavelength of light.

Changes in the ellipsometric angle $\Delta$ are given by the difference in $L$ parallel and perpendicular to the plane of incidence.

$$
\delta \Delta=-i\left(\delta L_{p}-\delta L_{s}\right)
$$

The parallel component reads

$$
-i \delta L_{p}=\frac{4 \pi}{\lambda} \cos \varphi \frac{\delta \gamma_{t}}{1-\epsilon_{2}}\left(1+\frac{1-\epsilon_{2} \delta \gamma_{n} / \delta \gamma_{t}}{\cot ^{2} \varphi-1 / \epsilon_{2}}\right)
$$

whereas the perpendicular component is given by

$$
-i \delta L_{s}=\frac{4 \pi}{\lambda} \cos \varphi \frac{\delta \gamma_{t}}{1-\epsilon_{2}}
$$

This model reflects the optical response at the molecular level, but the model suffers from its complexity and high number of parameters, the values of which can only be assumed. Since the macroscopic ansatz resulting in eqn. (54) comes to similar results with a properly chosen dependence of the refractive index of the layer on the adsorbed amount, the use of the microscopic model is not common in practical applications. In the following section we will discuss some representative examples. 


\section{Adsorption layers of soluble surfactants}

\subsection{Importance of purification}

Due to the peculiarities of surfactant synthesis, many surfactants contain trace impurities of higher surface activity than the main component. These trace impurities do not influence most bulk properties. However, at the surface they are enriched and impurities may even dominate the properties of the interface. This behaviour was first recognized by Mysels [15] and a purification scheme using foam fractionation was proposed [16]. A detailed discussion on artifacts caused by impurities can be found in [17].

In studies performed in our lab, we use a fully automated purification device developed by Lunkenheimer et. al. which ensures a complete removal of these unwanted trace impurities [18]. The aqueous stock solution undergoes numerous of purification cycles consisting of a) compression of the surface layer, b) its removal with the aid of a capillary, c) dilation to an increased surface and d) formation of a new adsorption layer. At the end of each cycle the surface tension $\sigma_{e}$ is measured. The solution is referred to as surface chemically pure grade if $\sigma_{e}$ remains constant in between subsequent cycles. Quite frequently more than 300 cycles and a total time of several days are required to achieve the desired state. The sample preparation is time consuming and tedious but mandatory for the investigation of equilibrium properties of adsorption layers of soluble surfactants at the air-liquid interface.

\subsection{Physicochemical properties of our model systems}

In the following we discuss the interfacial properties of two related amphiphiles, the cationic amphiphile 1-dodecyl-4-dimethylaminopyridinium bromide, C12-DMP, and the closely related nonionic 2-(4'dimethylaminopyridinio)-dodecanoate, C12-DMP betaine. The corresponding chemical structures are depicted in fig. 7 together with their equilibrium surface tension isotherms. The synthesis is described in [19].

Both components are classical amphiphiles and resemble all common features such as the existence of a critical micelle concentration $\mathrm{cmc}$. The members of the homologous series of the alkyl-dimethylaminopyridinium bromide are strong electrolytes and follow the pre- 
dictions of Debye-Hückel theory as experimentally verified by conductivity measurements. For solubility reasons we used 1-butyl-4-dimethylaminopyridinium bromide instead of the $C_{12}$ representative of the homologous series which gave us experimental access to a wider concentration range. Debye-Hückel predicts a proportionality of the activity coefficient to the square root of the ionic strength for aqueous solutions of 1:1 electrolytes which is indeed observed in our experiment.
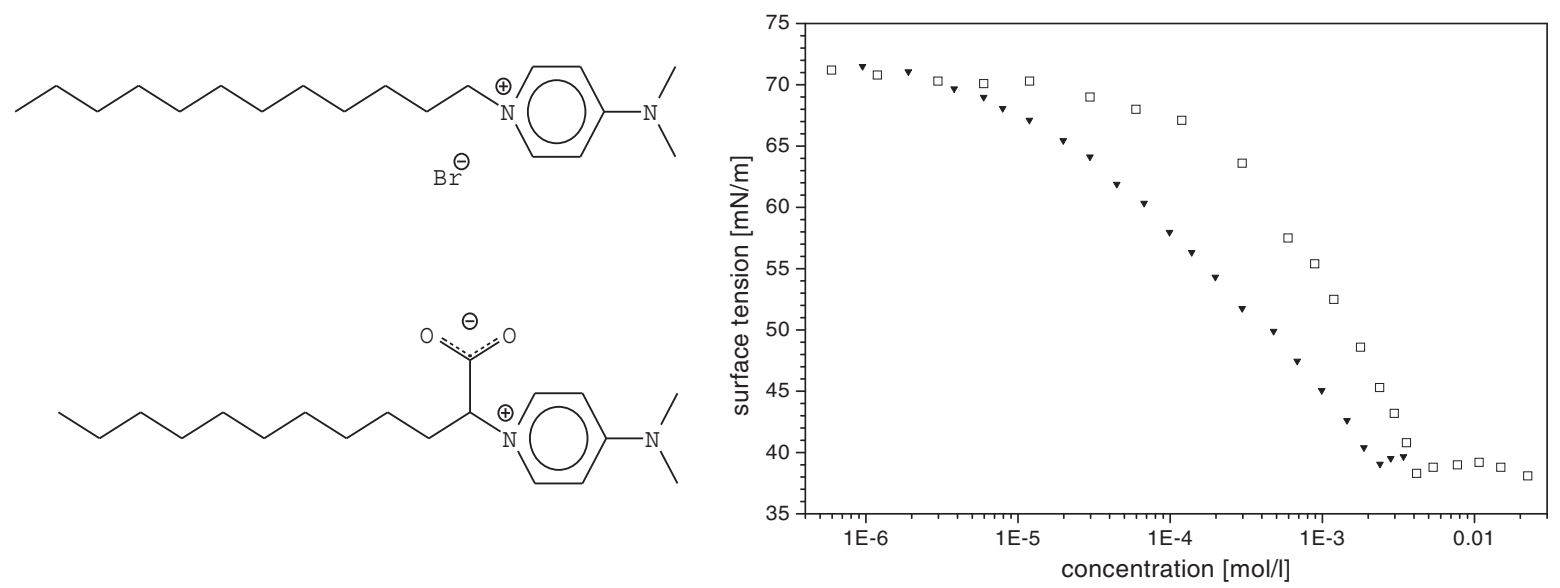

Figure 7: Chemical structures of the cationic amphiphile 1-dodecyl-4-dimethylaminopyridinium bromide, C12-DMP bromide, and the closely related nonionic 2-(4'dimethylaminopyridinio)-dodecanoate, C12-DMP betaine. The equilibrium surface tension $\sigma_{e}$ of a purified aqueous solution of the ionic C12-DMP bromide(squares) and the nonionic C12-DMP betaine(triangles) as a function of the bulk concentration $c_{o}$. (redrawn from $\left.[20]\right)$

\subsection{Adsorption layer of a nonionic surfactant}

The ellipsometric isotherm $\Delta-\Delta_{0}$ of the nonionic amhiphile C12-DMP betaine is shown in Fig. 8. It decreases in a monotoneous fashion and reaches a limiting value at higher concentration. In order to assess its physical meaning we compare $\Delta-\Delta_{0}$ to the surface excess retrieved from the surface tension isotherm Fig. 7. According to Gibbs' fundamental law:

$$
\Gamma=-\frac{1}{m R T} \cdot \frac{\mathrm{d} \sigma_{e}}{\mathrm{~d} \ln a} \approx-\frac{1}{m R T} \cdot \frac{\mathrm{d} \sigma_{e}}{\mathrm{~d} \ln c}
$$

the total surface excess $\Gamma$ is given by the derivative of the surface tension isotherm $\sigma_{e}(c)$. 
$\Gamma$ is then compared to the changes of the ellipsometric quantity $\Delta-\Delta_{0}$. The inset of Fig. 8 presents the result. The surface excess as given by the slope of the surface tension isotherm is compared to the ellipsometric response at each concentration. Obviously the relation between both quantities can be described by a straight line. Hence, experimental evidence has been provided that ellipsometry measures indeed the surface excess of the adsorption layer of the soluble nonionic amphiphile. Since optics measures refractive indices which are not very sensitive to molecular details we anticipate that this holds for a wider class of materials.

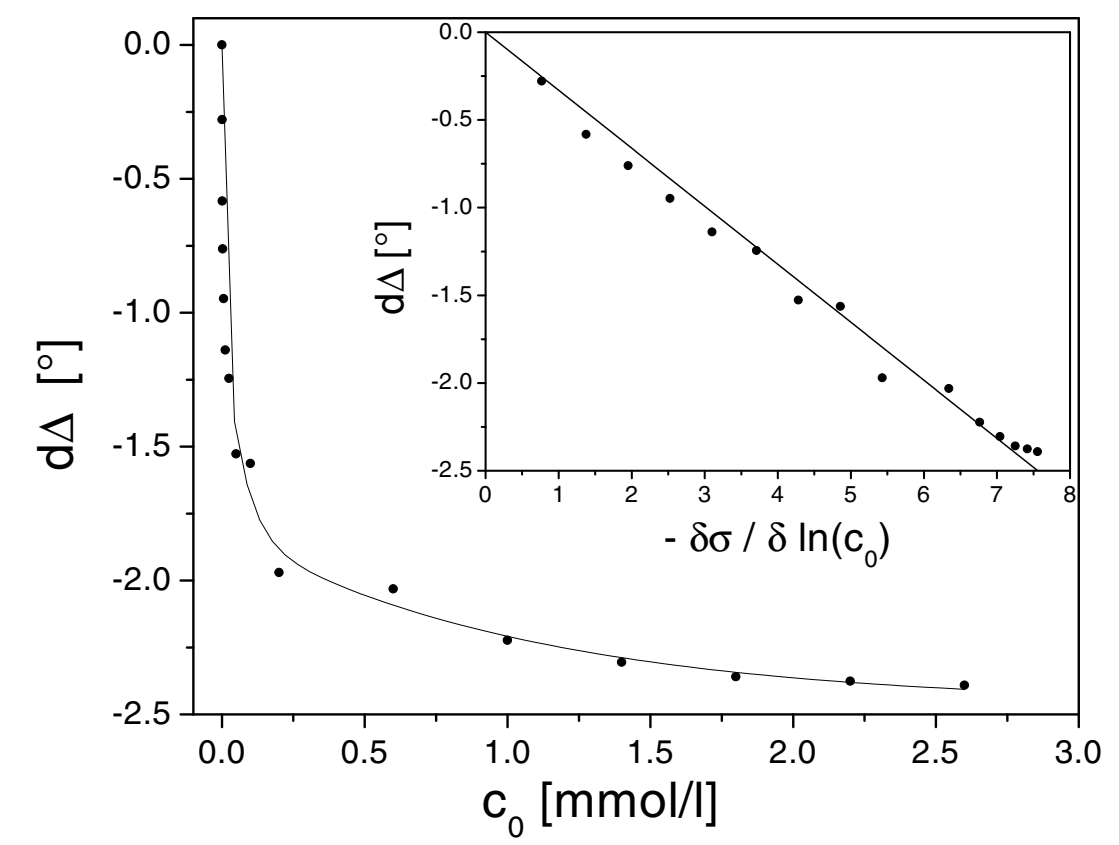

Figure 8: Ellipsometric isotherm of the nonionic C12-DMP betain. The ellipsometric isotherm decreases in a monotonic fashion and is proportional to the surface excess as can be seen in the inset, which compares the surface excess according to Gibbs and the ellipsometric response. (redrawn from [20])

\subsection{Ionic surfactant at the air-water interface}

In the previous section it was shown that in spite of the uncertainties from the mathematical point of view concerning the interpretation of eqn. (55) a proportionality between $\Delta$ and $\Gamma$ can be established by a comparison of thermodynamic and ellipsometric data for a nonionic surfactant at the air-water interface. In the following we will discuss a measure- 
ment carried out on adsorption layers of an ionic surfactant. It will be demonstrated that there are major differences between the two model systems.

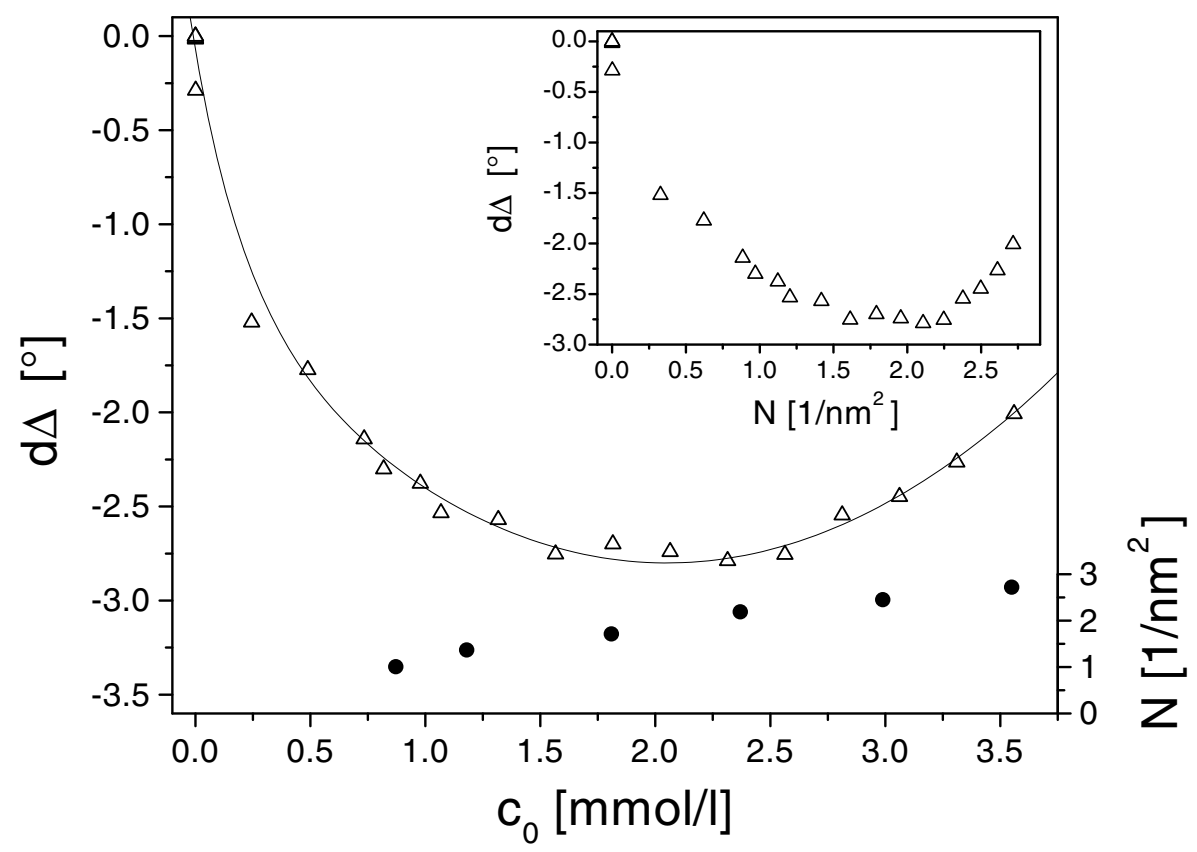

Figure 9: Characterization of the equilibrium properties of the adsorption layer by ellipsometry and Surface second harmonic generation, SHG. The SHG-signal $\sqrt{I^{2 \omega}(P=45, A=90)}$ (circles) is proportional to the surface coverage and increases monotonously with the bulk concentration. The ellipsometric quantity $d \Delta=\Delta-\Delta_{0}$ (triangles) shows an extremum at an intermediate concentration far below the $c m c$. The inset clearly shows the nonmonotonic dependency of $d \Delta$ on the adsorbed amount. (redrawn from [20])

Fig. 9 shows the ellipsometric isotherm $\Delta-\Delta_{0}$ (triangles) of the cationic surfactant C12DMP bromide. A pronounced non-monotonic behaviour is shown with an extremum at an intermidiate concentration far below the $\mathrm{cmc}$. Also shown is the number density of amphiphiles adsorbed to the interface (circles) as determined by Surface second harmonic generation (SHG). At these bulk concentrations the measured number density equals the surface excess $\Gamma$. SHG reveals a monotoneous increase in the surface excess in qualitative agreement to a thermodynamic analysis within the Gibb's framework. The data also clearly prove that the ellipsometric quantity need not be proportional to the adsorbed amount for a soluble ionic surfactant. What causes the nonmonotonous behaviour and how can it be understood?

In the following we discuss some possible scenarios: 


\section{Scenario 1: Filling up the adsorption layer}

The adsorption layer is described as an isotropic optical layer of constant thickness with a refractive index $n_{\text {layer }}$ which depends on the surface coverage [21]. Within this model there are two distinct surface concentrations which lead to a vanishing $d \Delta=\Delta-\Delta_{0}=0^{\circ}$. At very low coverage the refractive index of the layer matches the one of air $n_{\text {layer }}=n_{\text {air }}=1$ and at an intermediate surface coverage the surface layer adopts the very same refractive index as the water bulk phase $n_{\text {layer }}=n_{\text {water }}=1.332$. Consequently there has to be an extremum in between. The resulting $d \Delta\left(n_{\text {layer }}\right)$-curve is depicted in Fig. 10 . The geometrical dimension of the molecules has been used as thickness of the layer.

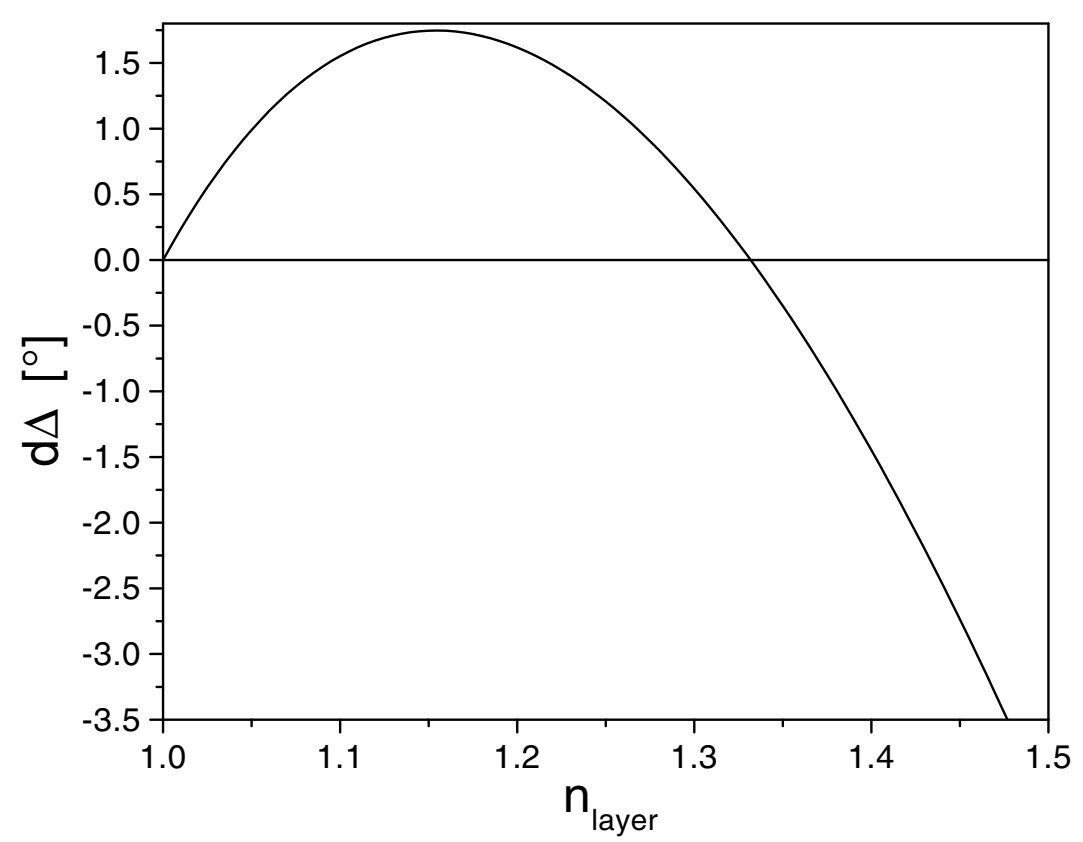

Figure 10: Simulation of the effect of a changing refractive index of a layer of constant thickness $h=2.1 \mathrm{~nm}$ on the ellipsometric signal $d \Delta$.

Obviously this scenario is not suitable to explain the measurement, the model predicts even a wrong sign of $d \Delta_{\max } \approx 1.75^{\circ}$ ! The experimental data require that $n_{\text {layer }}>n_{\text {water }}$ at all concentration. This means, water instead of air is the effective environment of this particular amphiphile within the adsorption layer. 


\section{Scenario 2: Effect of anisotropy}

The following provides an estimation if changes in the molecular orientation may be responsible for the surprising features in the ellipsometric isotherm. The following assumptions were made in order to estimate an upper limit for this effect:

- The optical model is that of an uniaxial layer with the optical axis normal to the interface. The molecular arrangement is $C_{\infty V}$ which has been experimentally verified for the headgroups by polarization dependent SHG measurements.

- The thickness of the adsorbed layer and the mean tilt angle of the molecules within the layer change with their number density $N$ at the interface. Within the investigated number density range the thickness increases from $1 \mathrm{~nm}$ to $1.9 \mathrm{~nm}$ proportional to the cosine of the tilt angle which is assumed to change from around $70^{\circ}$ to $40^{\circ}$.

- The whole molecule, including the head group, is assumed to change its tilt angle. The molecules are assumed to be all-trans and perfectly aligned which would yield the maximum possible change in anisotropy.

- The refractive index for an E-vector along the length axis of the molecule is $n_{\text {axis }}=$ 1.56, while the refractive index for an E-vector perpendicular to the long axis of the molecule is $n_{\text {perp }}=1.48$. Both refractive indices have been taken from Riegler et.al. [22] and rely on a combination of X-ray reflection data with ellipsometric measurements of monolayers of behenic acid at the air-water interface for a densely packed and perfectly oriented apmphiphiles. In our case the actual refractive indices will depend on the prevailing volume concentration and the data are therefore an upper limit.

The calculated $d \Delta(N)$ versus density-curve is plotted in Fig. 11. Obviously a change of the molecular tilt leads to changes in $d \Delta$, but cannot account for the measured pronounced extremum. In addition it is impossible to reach $d \Delta=-2.77^{\circ}$, which is the minimum of the measured curve, with reasonable parameters for the anisotropic layer. An increase in anisotropy has the same impact on the ellipsometric measurements as a reduction of 


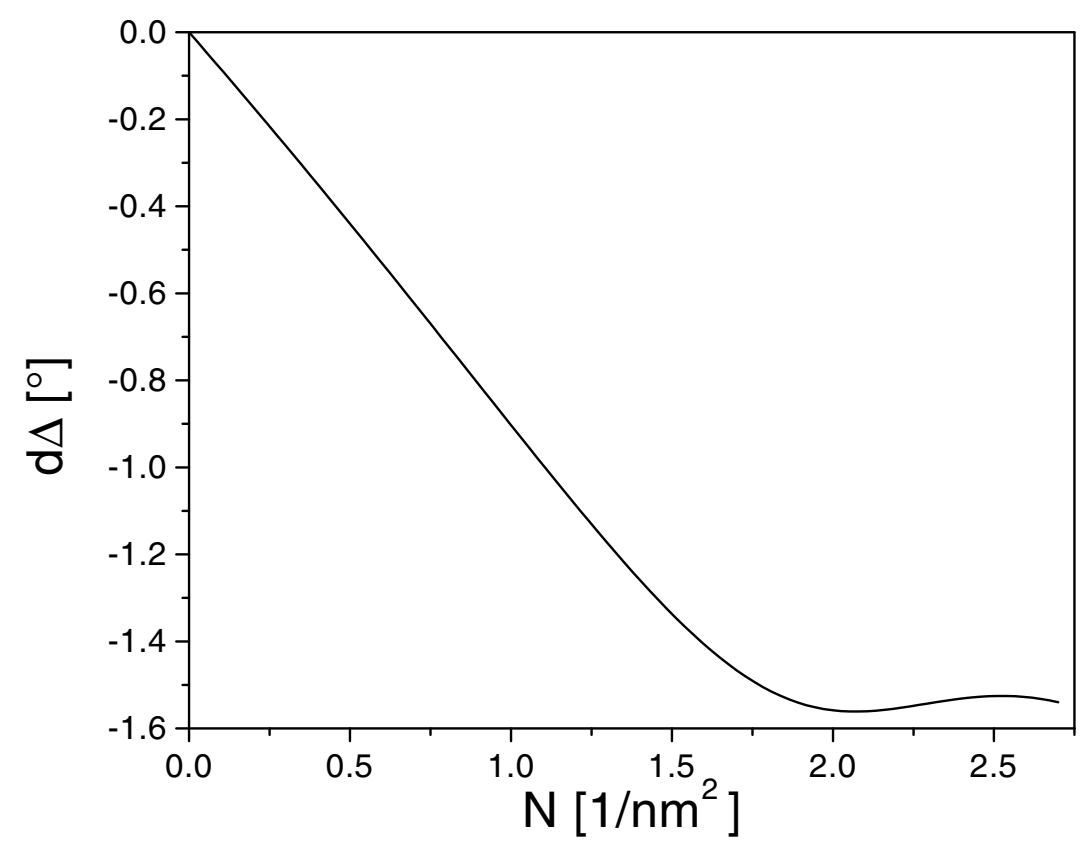

Figure 11: Investigation of the impact of anisotropy on the ellipsometric signal. The ellipsometric signal $d \Delta$ calculated in dependence of the number density of adsorbed molecules N. A molecular length of 2.1 $\mathrm{nm}$ and a refractive index of $n_{\text {axis }}=1.56$ for an E-vector along and of $n_{\text {perp }}=1.48$ for an E-vector perpendicular to the molecular axis were used. The layer thickness $(1.0 \mathrm{~nm}$ to $1.9 \mathrm{~nm})$, the tilt angle $\left(70^{\circ}\right.$ down to $\left.40^{\circ}\right), n_{s}$ and $n_{p}$ were all assumed to be dependent on the surface coverage.

the layer thickness; hence, the only way to get nearer to $d \Delta=-2.77^{\circ}$ is to diminish the anisotropy!

At this point we would like to point out that the assumptions made for this simulation are highly exaggerating the effect of the anisotropy. The SHG measurements reveal that the heads do not change their tilt angle at all. In other words that only the tails may change their tilt, which results in an even thinner anisotropic layer and therefore a smaller effect. Additionally the tails are certainly neither all-trans nor perfectly oriented even at the highest coverage measured $0.37 \mathrm{~nm}^{2} /$ molecule. For these reasons Fig. 11 represents an upper limit of the impact of anisotropy. The real effect is by far smaller for the surface coverages encountered here, which means that anisotropy cannot account for the surprising feature of the ellipsometric isotherm. 


\section{Scenario 3: Changing the counterion distribution}

Ellipsometry probes the complete interfacial architecture. The reflected light is generated within the transition region between the two adjacent bulk phases, air and aqueous surfactant solution. The electric double layer has to be explicetely considered. Hence, changes in the ion distribution at higher concentrations may cause the observed feature in the ellipsometric isotherm.

The classical model of a charged double layer has been developed by Gouy, Chapman and Stern. The interface is described by two distinct regions: a compact layer consisting of the positively charged adsorbed amphiphiles with some directly adsorbed counterions and a diffuse layer of counterions.

The ion distribution within the diffuse layer is given by the solution of the Poisson equation which relates the divergence of the gradient of the electric potential $\Phi$ to the charge density $\rho$ at that point (see for instance $[23,24]$ ):

$$
\operatorname{div} \operatorname{grad} \Phi \Rightarrow \Phi=\frac{\rho}{\epsilon_{o} \epsilon_{r}}
$$

The compact layer is positively charged and in contact with an electrolyte solution which forms a diffuse layer of charges. The ion concentration distribution within the electrical potential $\Phi$ obeys Boltzmann:

$$
c^{-}=c_{o}^{-} e^{\frac{z^{-} e \Phi}{k_{B} T}} \quad c^{+}=c_{o}^{+} e^{-\frac{z^{+} e \Phi}{k_{B} T}}
$$

where $z^{-}$and $z^{+}$are the valencies of the anions and cations respectively. For a symmetric electrolyte solution $\left(-z^{-}=z^{+}=z\right.$ and $c_{o}^{-}=c_{o}^{+}=c_{o}$ ) eqn.(84) leads to a net charge of the ion cloud of

$$
\rho=z e\left(c^{+}-c^{-}\right)=-2 c_{o} z e \sinh \frac{z e \Phi}{k_{B} T}
$$

The combination of eqn.(85) with the Poisson eqn.(83) yields a differential equation in the electric potential $\Phi$. In our case the potential is only a function of the normal coordinate to the surface $x$. It is convenient to define a reduced potential 


$$
y=\frac{z e \Phi}{k_{B} T} \quad y_{o}=\frac{z e \Phi_{o}}{k_{B} T}
$$

which further simplifies the equations and results in

$$
\frac{d^{2} y}{d x^{2}}=\frac{2 c_{o} z^{2} e^{2}}{k_{B} T \epsilon_{o} \epsilon_{r}} \sinh y=\kappa^{2} \sinh y
$$

The integration of equation (87) with the boundary conditions $(y=0$ and $d y / d x=0)$ for $x=\infty$ and $y=y_{o}$ at $x=0$ lead to the Gouy-Chapmann solution of the reduced potential within the diffuse layer:

$$
e^{y / 2}=\frac{e^{y_{o} / 2}+1+\left(e^{y_{o} / 2}-1\right) e^{-\kappa x}}{e^{y_{o} / 2}+1-\left(e^{y_{o} / 2}-1\right) e^{-\kappa x}}
$$

Hence, knowing the charge of the compact layer the ion distribution can be modelled. The crux of Stern's treatment is the estimation to which extent ions enter the compact layer and reduce the surface potential. The ion distribution between compact and diffuse layer cannot be easily experimentally established, for example surface potential measurements cannot measure this distribution.

The optical analysis requires the translation of the prevailing distribution of molecules and ions within the interphase into a corresponding refractive index profile. The reflectivity coefficients can then be calculated on the basis the previously discussed numerical algorithms for stratified media. Two elements dominate the refractive index profile and hence the reflectivity properties, the topmost monolayer of the amphiphile and the distribution of ions within the diffuse layer. The excess of ions within the diffuse layer leads to a slightly elevated refractive index as compared to the bulk of the aqueous surfactant solution. The typical dimensions of the diffuse layer are on the order of $10 \mathrm{~nm}$ and this fairly wide extension leads to a profound impact on $\Delta$, since the topmost monolayer has a normal extension of only $2 \mathrm{~nm}$. The solution of the potential $\Phi$ plugged into equation (85) yields the distribution of anions and cations within the diffuse layer. The prevailing charge distribution is determined by the surface charge of the topmost monolayer. The refractive index profile is then determined by the ion distribution by a multiplication with 
the refractive index increment $d n / d c$ which has been independently measured with an Abbe refractometer.
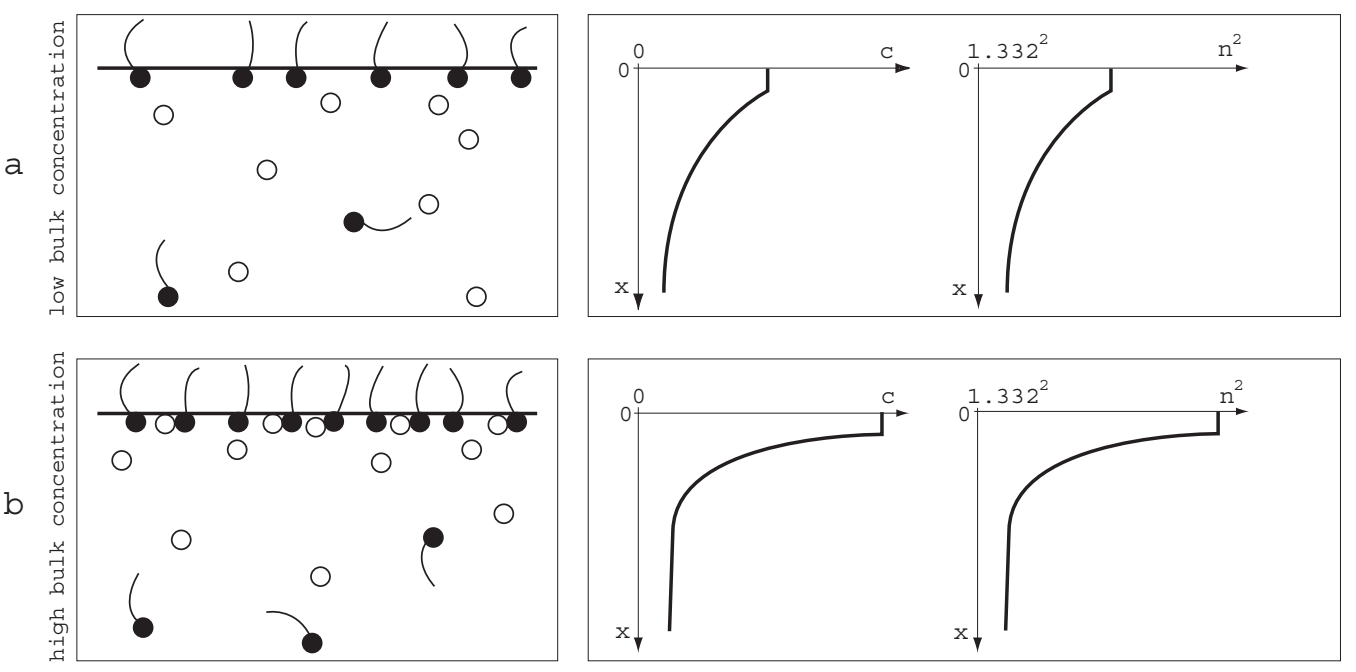

Figure 12: The interphase of a soluble cationic surfactant at the air-water interface at low (a) and high (b) bulk concentration. It consists of a charged topmost cationic monolayer, a diffuse layer of counterions and at higher concentrations a compact layer of directly adsorbed counterions. The charge density of the topmost monolayer reduced by the charge of the inner Stern layer determines the ion distribution within the diffuse layer. The prevailing ion distribution is given by solution of the nonlinear Poisson-Boltzmann equation. The excess of ions can be readily translated in a corresponding refractive index profile. The profile determines the reflectivity properties. Ellipsometric data modeled within this framework allow an estimation of the extent to which ions enter the compact layer.

Fig. 12 sketches the model for the interface. At lower surface coverage most of the ions are spread out within the diffuse layer leading to a pronounced refractive index profile. The situation is sketched in Fig. 12a. At higher concentration some ions enter the adsorption layer and form ion pairs with the headgroups accounted with a lower $d n / d c$-value as compared to the bulk phase. The formation of this Stern layer effectively reduces the surface charge and as a consequence the extension and the magnitude of the refractive index profile of the diffuse layer decrease. This situation is sketched in Fig. 12b.

This model has been used for monitoring the formation of the Stern layer. The ion distribution within the diffuse layer is determined by the total effective charge density at the interface. The SHG measurements yield the number density and charge density 


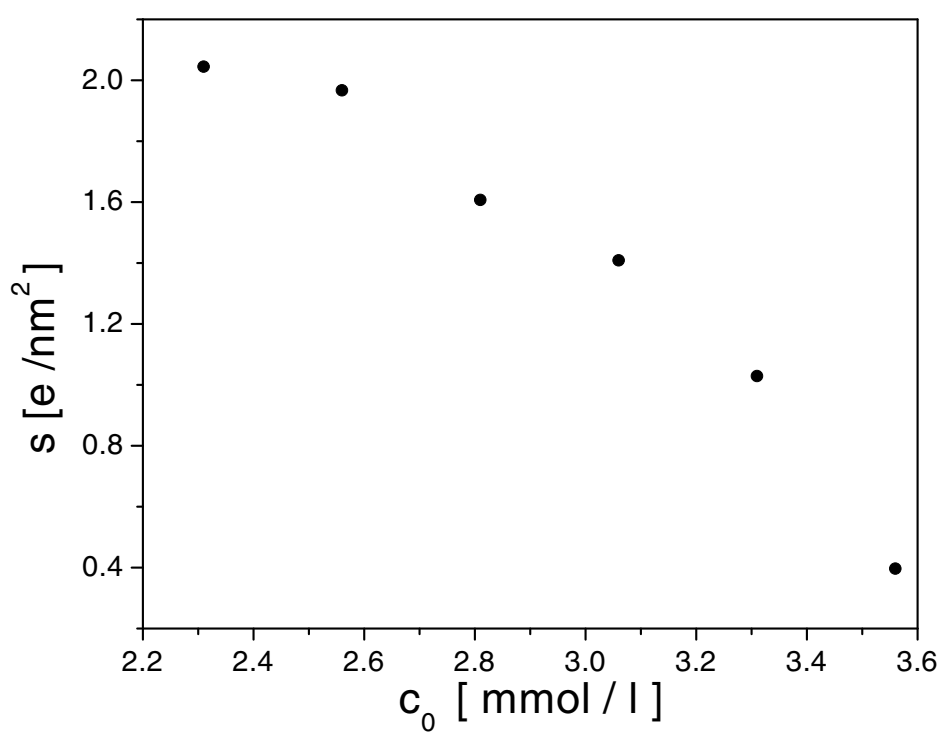

Figure 13: The charge density of the compact Stern layer has been retrieved by optical means. The surface charge refers to the number density of the cationic amphiphile reduced by the number of directly bound ions within the Stern layer.

produced by the cationic amphiphilic monolayer. The effective charge density at the interface is given by this value reduced by the number density of counterions within the Stern layer. The corresponding refractive index of Stern and topmost monolayer is given by an effective medium approach. Hence the optical properties of the monolayer are known by independent means. Furthermore the refractive index profile of the diffuse layer is given once the effective charge density at the interface is known. We used the experimental data in order to retrieve the corresponding effective surface charge density. The results are depicted in Fig. 13 starting in the vicinity of the extremum of the ellipsometric isotherm. Hence, with this model we are able to monitor by purely optical means the extent to which ions enter the compact Stern layer.

In short, ellipsometry applied to adsorption layers of ionic soluble surfactants does not measure the surface excess. The ellipsometric signal may show a non-monotonic behaviour which is caused by a redistribution of the ions between compact and diffuse layer. The data analysis within the classical model of a charged double layer yields an estimate of the prevailing ion distribution.

Ellipsometry applied to adsorption layers of nonionic surfactant directly yields the surface 
excess. It is therefore a valuable alternative to surface tension measurements, especially if one considers rheological properties which require higher derivatives of the surface tension isotherm.

\subsection{Kinetics of absorption}

Rapidly expanding liquid surfaces occur in many technical processes such as foam formation, spraying or painting, they are also omnipresent in nature, i.e. the oxygen exchange in the lung. Surfactants play a crucial role in these processes. They stabilize the new surfaces by a reduction of the surface tension. Inhomogeneities in surface coverage lead to gradients in surface tension which in turn have a strong impact on bulk hydrodynamic flow, a phenomenon also known as Marangoni flow. The overall dynamic behaviour is very complex and despite many efforts far from understood [25]. The investigation requires time resolution within the $m s$ regime putting severe limitations on the choice of the experimental technique. The maximum bubble pressure method [25] is most often used to perform these types of studies but ellipsometry offers an interesting alternative to supplement these investigations. This method relies on surface tension measurements and the surface coverage is obtained using the equilibrium surface isotherm. The underlying assumption is that the surface is locally at equilibrium, however, in the time regime of interest this may not be the case. As outlined in the theoretical section ellipsometry measures directly the surface coverage for nonionic surfactants and provides in addition the requested time resolution. The challenging part is the design of an experiment with precisely defined boundary conditions required for modeling the underlying physics leading to the observed kinetic behavior. The most promising approaches produce an interface with a non-equilibrium surface coverage, and maintain that surface coverage through the flow of fluid in and out of the control volume. To satisfy the requirement of well-defined boundary conditions, at the beginning of the flow a freshly formed surface must be created. Several arrangements have been suggested such as the inclined plate [25], the overflowing cylinder [26] or the use of a jet [27]. In all these approaches the surface has a defined age at a given spot, hence the desired dynamic picture of the surfactant adsorption is obtained by moving the sample relative to the light beam. 


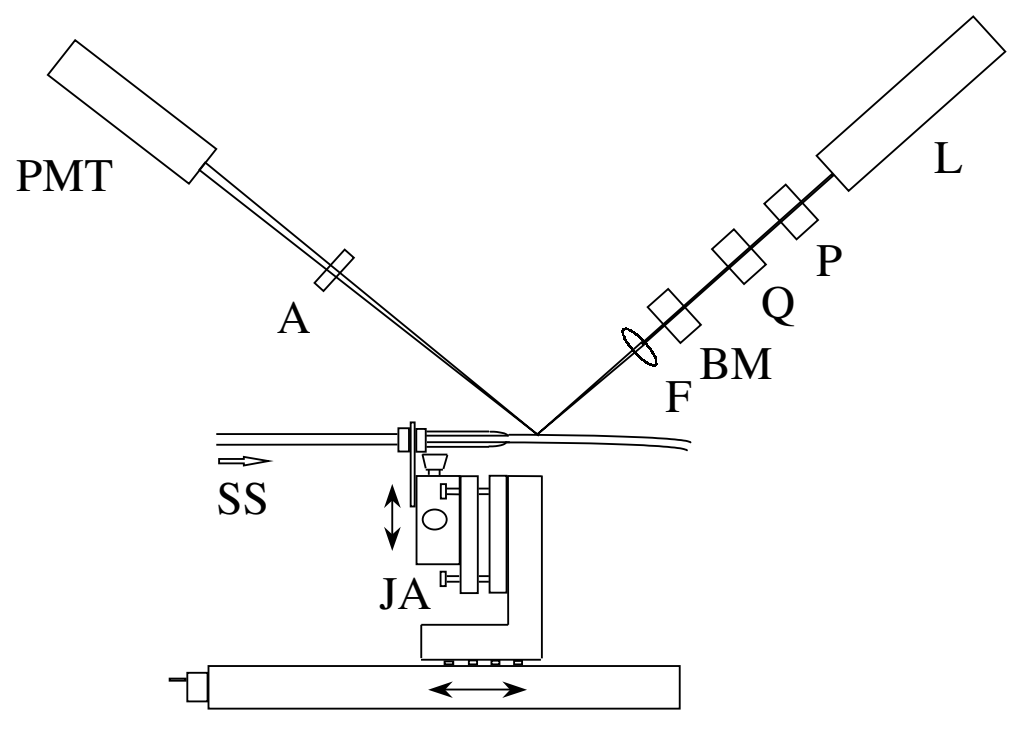

Figure 14: Setup for an ellipsometric measurement on a jet. The distance between laser spot and nozzle determines the age of the surface. (redrawn from Langmuir 15, 7530-7533 (1999))

The overflowing cylinder with its flat surface is very well suited for optical reflection technique. Water is pumped vertically trough a cylinder and allowed to flow over the rim in a radial flow pattern. This experiment covers the time regime of $0.1-1 s$ and has been successfully used by Penfold et.al. [28] in neutron reflectometry and ellipsometric experiments. A precision in the determination of the dynamic surface excess of $2 \cdot 10^{-8} \mathrm{~mol} / \mathrm{m}^{2}$ has been demonstrated. Neutron reflectivity enabled the calibration of the ellipsometric data which is important to avoid artifacts caused by changes in the ion distribution of ionic surfactants. The more interesting time range of $1-20 \mathrm{~ms}$ is covered by jet techniques. The surfactant solution is pumped with a high speed through a nozzle. An ellipsometric experiment performed on a jet is quite demanding and many subtle problems are caused by the curved surface acting as a cylindrical lens. A recent feasibility study of Hutchison et.al. demonstrates that these problems can be overcome [29]. The adsorption of a cationic surfactant was monitored and the time dependence was found to obeye the WardTordai equation [30] providing evidence for a diffusion controlled adsorption kinetics of the surfactant in this time regime. These studies suffer from a simultaneous measurement of the flow field which is required to assess Maragoni currents induced by surface tension gradients. 


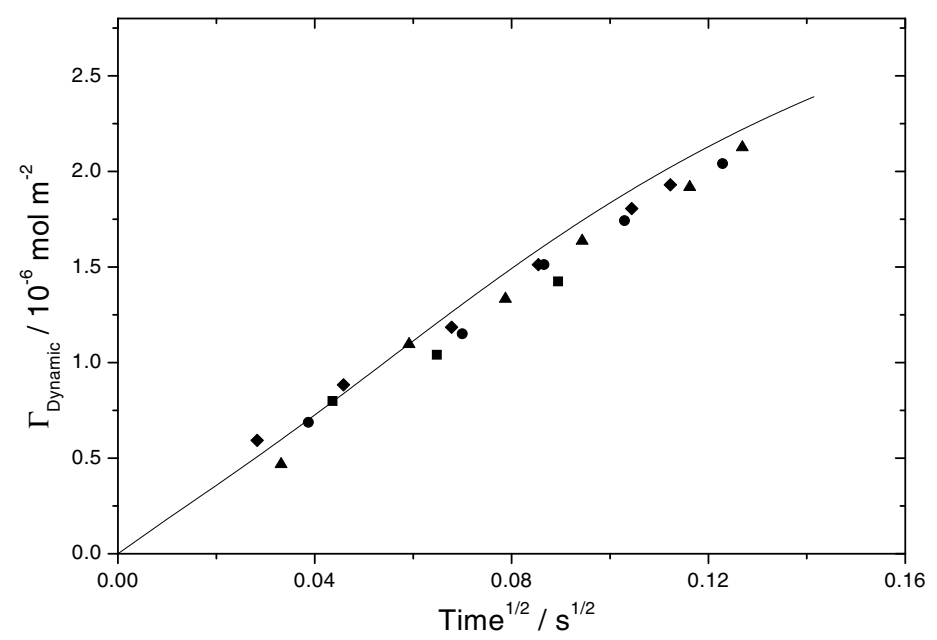

Figure 15: Surface coverage measured with the setup sketched in Fig. 14. (redrawn from Langmuir 15, 7530-7533 (1999))

\section{$9 \quad$ Principle of imaging ellipsometry}

Some surfaces under investigation are laterally inhomogeneous on a micrometer scale due to variations in the thickness or surface composition or due to changes in the orientational order of the molecules at the interface. In this case the lateral inhomogeneity is imparted to the properties of the reflected light. The most well-known imaging technique for a visualization of these features is Brewster Angle Microscopy BAM [31, 34] which has been successfully employed for characterizing the phase diagrams and the morphology of Langmuir films. BAM is based on the principle that the reflectivity of the $\hat{p}$-polarized light is zero at the Brewster angle. Any modification of the Brewster conditions, as for instance the presence of a single monolayer, modifies the reflectivity. A decisive advantage of this method vs. fluorescence microscopy [35] is that fluorescent labelling is not required. Also the internal structures of domains can be assessed.

Ellipsometry can also be extended to an imaging technique which offers a wider field of applications [9]. In contrast to BAM, imaging is not bound to the existence of a Brewster angle and can even be employed for the investigation of monolayers on highly reflecting supports.

Fig. (16a) provides a Nullellipsometric image of a self assembled monolayer of (Dimethyl- 

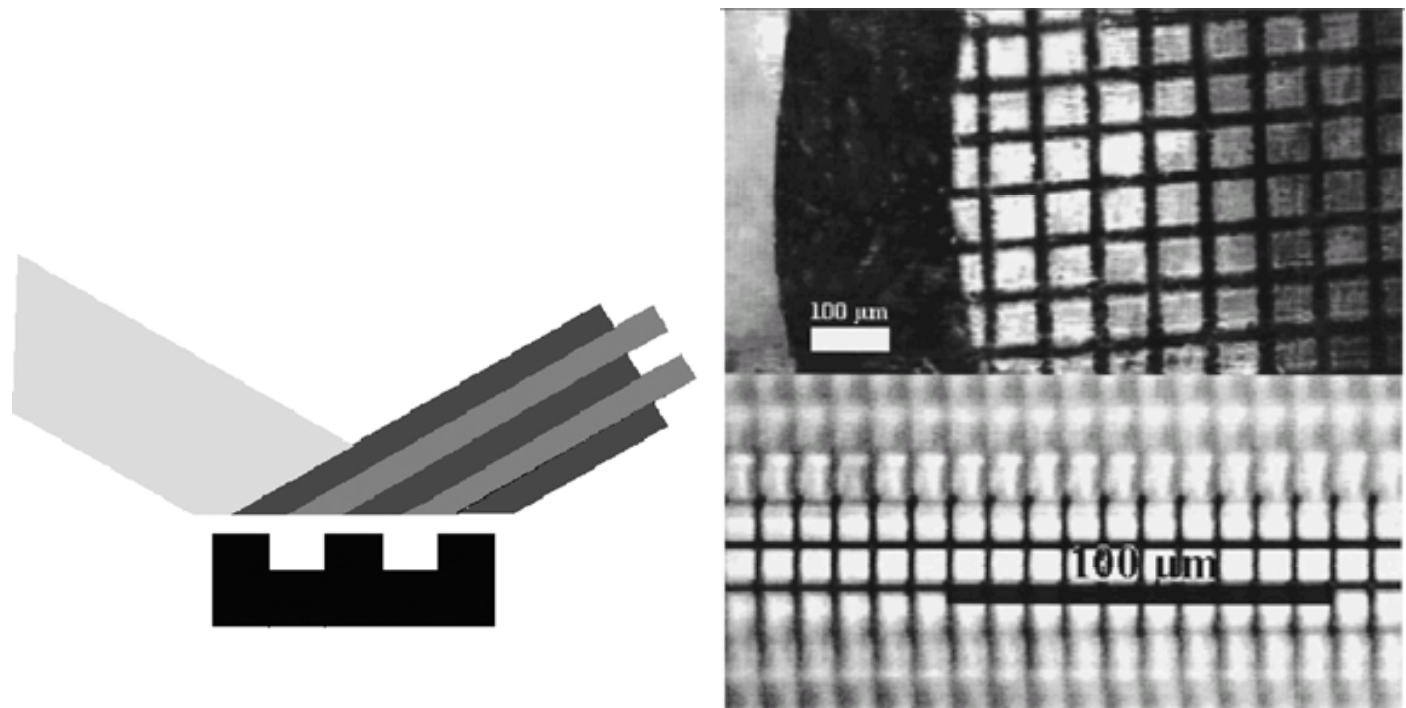

Figure 16: The lateral inhomogeneitity is transformed in the state of polarization. a) Nullellipsometric image of a self assembled monolayer on a silicon wafer. The monolayer was patterned with the aid of a mask and UV-light. The difference in the thickness between the dark and bright regions is $0.8 \mathrm{~nm} \mathrm{~b}$ ) At high magnification problems arise due to the limited depth of field. The width of the bars are $1.9 \mu m$, the mesh size is $10 \mu m$ and imaging is performed at an angle of $53^{\circ}$

chlorosilyl)-2-(p,m-chloromethylphenyl)ethan on silicon. The monolayer was photochemically pattererned using UV-irradiation and an electron microscopy grid as a mask. The difference in the thickness between dark and bright regions is $0.8 \mathrm{~nm}$ demonstrating the high vertical resolution of this technique. At a higher magnification there are certain problems arising from the limitation of the depth-of-field.

\subsection{Depth of field problem}

All the above mentioned imaging techniques work under an oblique angle of incidence and as a result some peculiarities exist. Imaging under an oblique angle of incidence imposes certain restrictions on the diameter and working distance of the objective. As a result the resolution is limited to about $1-3 \mu \mathrm{m}$. A discussion of this issue can be found in [32].

The depth of field, $t$, of an objective is given by the numerical aperture A, the refractive index $n$ of the ambient medium, and the wavelength $\lambda$ according to [33]:

$$
t=\frac{n \lambda}{A^{2}}
$$


Depending on the angle of incidence, $\varphi$, and the numerical aperture, A, only a region of the illuminated part of the sample is in focus. This region is of the order of $1-50 \mu \mathrm{m}$. This problem is illustrated in Figure (16b). The size of a bar is $1.9 \mu m$, the mesh size is $10 \mu \mathrm{m}$ and imaging was performed at $\varphi=53^{\circ}$.

The depth-of-field problem is a severe limitation at higher magnification. A straightforward solution consists of a scan of the objective [34]. Only the area in focus is recorded and the complete image is constructed by many stripes by imaging processing software. This procedure yields sharp images of static objects. At the oil-water or air-water interface it is only of limited use due to the prevailing convection.

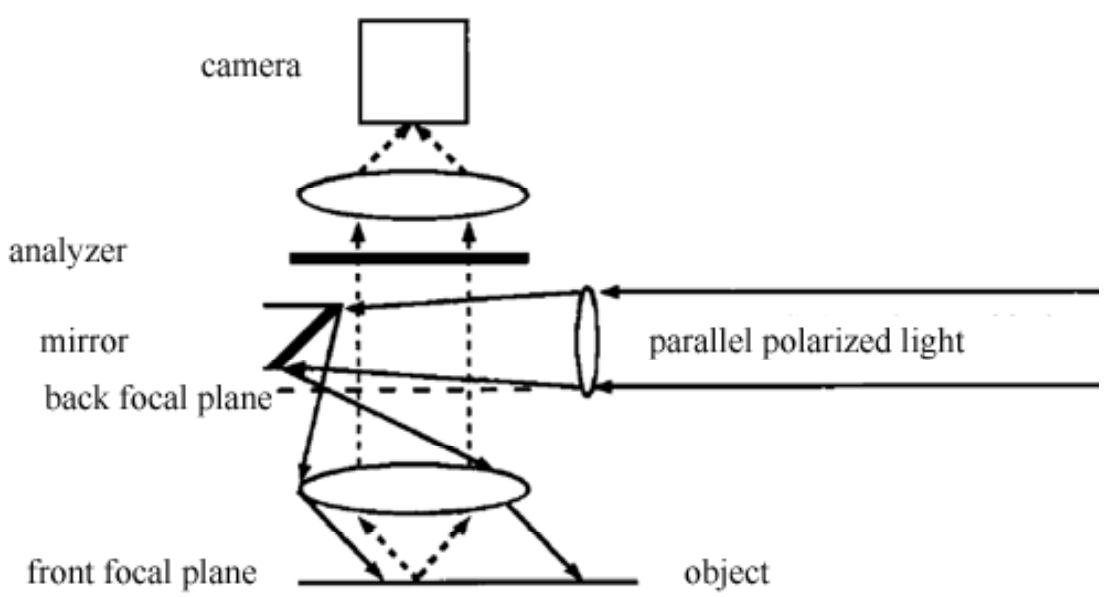

Figure 17: Principle of ellipsometric microscopy. Full arrows symbolise the light path of illumination, broken arrows stand for the observation, respectively. A parallel beam of polarised light is focussed into an off-axis spot in the back focal plane of the lens. In the front focal plane of the lens, where the object is located, this results in a parallel polarised beam of light hitting the object under a shallow angle of incidence. Light reflected by the object is collected by the lens, passes a motorised polarization analyzer and is focused onto a digital CCD camera. For clarity, several optical elements are omitted. (redrawn from $[36])$

An elegant approach which overcomes these difficulties was suggested in the group of Sackmann et al.. A parallel polarized laser beam was focused to an off axis spot in the back focal plane of a microscope objective of high numerical aperture. The sample which is located in the front plane is then illuminated by a parallel polarized beam of light incident on the sample under an oblique angle. The exact angle of incidence is controlled by the 
exact position of the laser focus in the back focal plane. The image is formed by the same objective used for illumination and the complete image is in focus [36].

The performance of this microscope is shown in Fig. 18. The sample consists of a photopatterned LB film with dimensions indicated in the figure. The image on the left hand side sketches the inner structure of the sample.

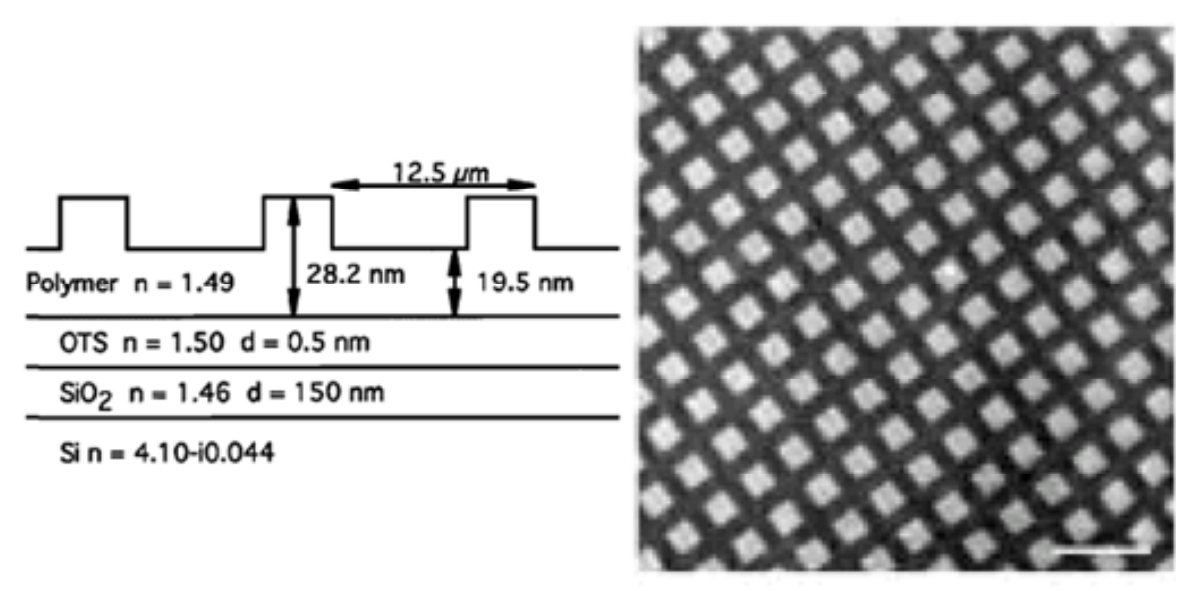

Figure 18: Schematic view of the micropatterned Langmuir-Blodgett film with all relevant dimensions; Ellipsometric microscopy images of the sample. The angle of incidence was $\varphi=55.8^{\circ}$. The gray scales from black to white represent a change in $\Delta$ of $120-130^{\circ}$. The scale bar has a length of $25 \mu \mathrm{m}$. (redrawn from [36])

Another elegant approach was suggested by Meunier et. al. who presented a custom made objective based on a modified Schwarzschild objective. Its optic axis is perpendicular to the studied layer and consequently the complete area is in focus. The design is ideally suited for dynamic investigations and the alignment is easier than for the previously discussed solution.

\subsection{Beyond the diffraction limit}

The high vertical resolution of ellipsometry is not matched by the achieved lateral resolution. The upper limit is given by diffraction and on the order of the wavelength of light. Recently an experiment has been described which overcomes the diffraction limit and allows a direct visualization of refractive index patterns with a much finer resolution 
[37]. The arrangement is based on a combination of Atomic Force Microscopy (AFM) and Ellipsometry and the AFM tip dimensions determines the resolution.

The sample of interest is mounted on the base of a prism. The ellipsometer is operated in the null mode and the total reflected light at the prism base is completely cancelled by the setting of the polarization optics. This null setting is kept constant during the course of the experiment. The metal AFM tip couples to the evanescent field and depending on the local optical properties of the sample the null setting is out of tune. The intensity at the detector is monitored while carrying out a topographic scan with a conventional Atomic Force Microscope. Thus two images are simultaneously generated: a topographic image by the AFM tip and an ellipsometric image which relates the intensity reading at the detector with the $\mathrm{x}, \mathrm{y}$ position of the tip. The latter contains information on refractive index inhomogenity. This technique allowed for instance a visualization of $10 \mathrm{~nm}$ CDTe particles within a polymer matrix. The topographic scan was not able to identify the particles within the spincoated polymer film. However, due to the differences in the refractive index it could be visualized by the ellipsometric image.

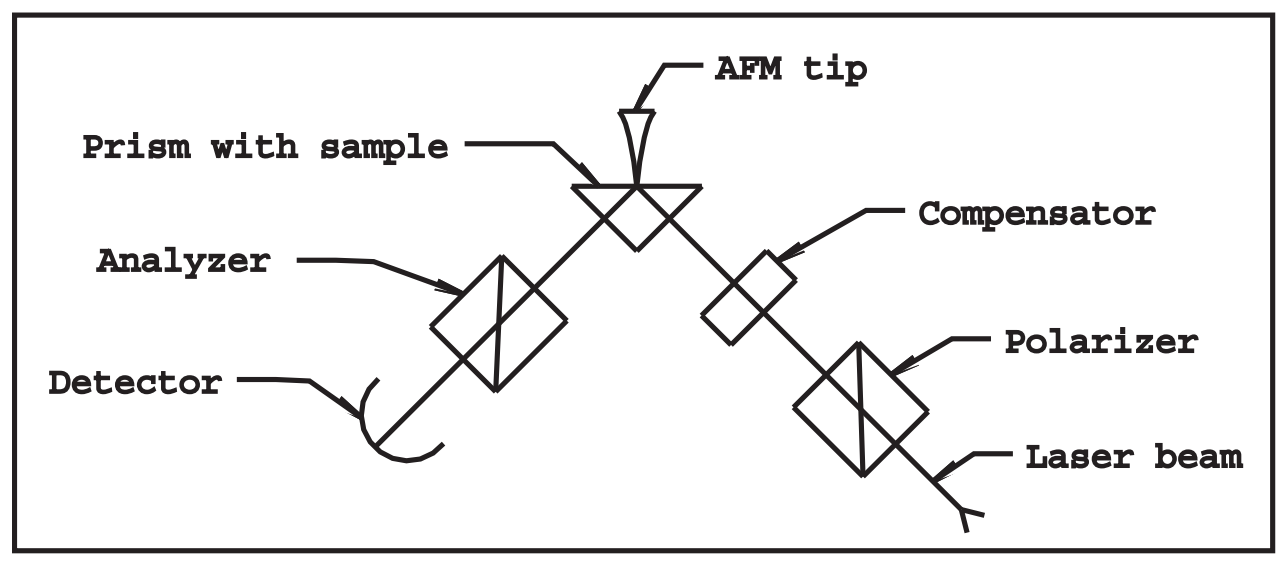

Figure 19: Sketch of the used apparatus 


\section{References}

[1] M. Born, Optik, Springer Verlag, New York, Heidelberg (1998)

[2] D. S. Kliger, J. W. Lewis, C. Randall, Polarized Light in optics and spectroscopy, Academic Press, Harcout Brace Javanovich Publishers, Boston (1990)

[3] R. M. Azzam, N.M. Bashara, Ellipsometry and Polarized Light, North Holland Publication, Amsterdam (1979)

[4] R.C. Jones, J. Opt. Soc. Am 16, 488 (1941)

[5] S.N. Jasperson, S.E. Schnatterly, Rev. Sci. Instr. 40 , 761 (1969)

[6] J. Lekner, Theory of Reflection, Martinus Nijhoff Publishers, Boston, (1987)

[7] T. Kull, T. Nylander, F. Tiberg, N. Wahlgren, Langmuir 13, 5141 (1997)

[8] R. Teppner, S. Bae, K. Haage, H. Motschmann, Langmuir 15, 7002 (1999)

[9] R. Reiter, H. Motschmann, H. Orendi, A. Nemetz, W. Knoll, Langmuir 8, 1784 (1992)

[10] C. Flueraru, S. Schrader, V. Zauls, H. Motschmann, Thin Solid Films 379, 15 (2000)

[11] H. Motschmann, M. Stamm, C. Toprakcioglu, Macromolecules 24, 3681 (1991)

[12] J. Lekner, P.J. Castle, Physica 101A, 89 (1980)

[13] M.J. Dignam, M. Moskovits, J. Chem. Soc. Faraday II 69, 56 (1973)

[14] M.J. Dignam, J. Fedyk, J. Phys. (Paris) 38, C5-57 (1977).

[15] P.H. Elworthy, K.J. Mysels, J. Coll. Int. Sci. , 331 (1966)

[16] K.J. Mysels, A. Florence, J. Coll. Int. Sci. 43, 577 (1973)

[17] K. Lunkenheimer, J. Coll. Int. Sci. 131, 580 (1989)

[18] K. Lunkenheimer, H.J. Pergande, H. Krüger, Rev. Sci. Instr. 58, 2313 (1987)

[19] K. Haage, H. Motschmann, S. Bae, E. Gründemann, Colloids and Surfaces (in press) 
[20] R. Teppner, K. Haage, D. Wantke , H. Motschmann, J.Phys.Chem.B 104, 11489 (2000)

[21] T. Pfohl, H. Möhwald, H. Riegler, Langmuir 14, 5285 (1998)

[22] M. Paudler, J. Ruths, H. Riegler, Langmuir 8, 184 (1992)

[23] D.F. Evans, H. Wennerström, The Colloidal Domain, VCH Publishers, New York (1994)

[24] A.W. Adamson, Physical Chemistry of Surfaces, Wiley \& Sons, New York (1993)

[25] S.S. Dukhin, G. Kretzschmar, R. Miller, Dynamics of Adsorption at Liquid Interfaces, Elsevier, Amsterdam (1995)

[26] D.J.M. Bergink-Martens, H.J. Bos, A. Prins, B.C. Schulte, J. Coll. Int. Sci. 138 (1990); D.J.M. Bergink-Martens, H.J. Bos, A. Prins, J. Coll. Int. Sci. 165, 221 (1994)

[27] B.A. Noskov, Adv. Colloid Interface Sci. 69, 63 (1996)

[28] S. Manning-Benson, S. R. W. Parker, C. D. Bain, J. Penfold, Langmuir 14, 990 (1998)

[29] J. Hutchison, D. Klenerman, S. Manning-Benson, C. Bain, Langmuir 15, 7530 (1999)

[30] A.F.H. Ward, L. Tordai, J. Chem. Phys. 14, 453 (1946)

[31] D. Hönig, D. Möbius, J. Phys. Chem 95, 4590 (1991)

[32] M. Harke, R. Teppner, O. Schulz, H. Orendi, H. Motschmann, Rev. Sci. Instrum. 68, 8, 3130 (1997)

[33] H. Riesenberg Handbook of Microscopy, VEB Verlag Technik, Berlin (1988)

[34] S. Henon, J. Meunier, Rev. Sci. Instr. 62, 936 (1991)

[35] M. Lösche, E. Sackmann, H. Möhwald, Ber. Bunsenges. Phys. Chem. 10, 848 (1983)

[36] K.R. Neumaier, G. Elender, E. Sackmann, R. Merkel, Europhysics Letters 49 (1), 14 (2000)

[37] P. Karageorgiev, H. Orendi, B. Stiller, L. Brehmer, Appl. Phys. Lett., (2001) in press 